\title{
Partial-Sum Queries in OLAP Data Cubes Using Covering Codes
}

\author{
Ching-Tien Ho, Member, IEEE, Jehoshua Bruck, Senior Member, IEEE, \\ and Rakesh Agrawal, Senior Member, IEEE
}

\begin{abstract}
A partial-sum query obtains the summation over a set of specified cells of a data cube. We establish a connection between the covering problem in the theory of error-correcting codes and the partial-sum problem and use this connection to devise algorithms for the partial-sum problem with efficient space-time trade-offs. For example, using our algorithms, with 44 percent additional storage, the query response time can be improved by about 12 percent; by roughly doubling the storage requirement, the query response time can be improved by about 34 percent.
\end{abstract}

Index Terms-Partial-sum query, covering code, error-correcting code, on-line analytical processing, data cube, multidimensional database, precomputation, query algorithm.

\section{INTRODUCTION}

$\mathrm{O}$ N-LinE Analytical Processing (OLAP) [7] allows companies to analyze aggregate databases built from their data warehouses. An increasingly popular data model for OLAP applications is the multidimensional database (MDDB) [18], also known as data cube [9]. To build an MDDB from a data warehouse, certain number of attributes are selected. Thus, each data record contains a value for each of these attributes. Some of these attributes are chosen as metrics of interest and are referred to as the measure attributes. The remaining attributes, say $d$ of them, are referred to as dimensions or the functional attributes. The measure attributes of all records with the same combination of functional attributes are combined (e.g., summed up) into an aggregate value. Thus, an MDDB can be viewed as a $d-$ dimensional array, indexed by the values of the $d$ functional attributes, whose cells contain the values of the measure attributes for the corresponding combination of functional attributes.

Consider a data cube from an insurance company as an example. Assume the data cube has four functional attributes (dimensions): age, time, state, and (insurance) type. Further assume that the domain of age is 1 to 100 , of time is 1Qtr87 to 4Qtr96 (four quarters per year and over 10 years), of state is the 50 states in the U.S., and of type is \{health, home, auto, life\}. The data cube will have $100 \times 40 \times 50 \times 4$ cells, with each cell containing the total revenue (the measure attribute) for the corresponding combination of age, time, state, and type, e.g., (35, 1Qtr96, California, auto).

We consider a class of queries, which we shall call partialsum queries, that sum over all selected cells of a data cube,

- C.-T. Ho and R. Agrawal are with IBM Almaden Research Center, 650 Harry Road, San Jose, CA 95120.

E-mail: \{ho, ragrawal\}@almaden.ibm.com.

- J. Bruck is with the California Institute of Technology, Mail Stop 136-93, Pasadena, CA 91125. E-mail: bruck@paradise.caltech.edu.

Manuscript received 29 Sept. 1997.

For information on obtaining reprints of this article, please send e-mail to: tc@computer.org, and reference IEEECS Log Number 105727. where selection is specified by providing a subset of values for some of the functional attributes. Partial-sum queries are frequent with respect to categorical attributes whose values do not have a natural ordering, although they can arise with respect to numeric attributes as well. Using the same example of an insurance data cube, a partial-sum query may obtain the total revenue from the states of California, Florida, Texas, and Arizona, for life and health insurances, and for 1Qtr94, 1Qtr95, and 1Qtr96. In an interactive exploration of data cube, which is the predominant OLAP application area, it is imperative to have a system with fast response time.

\subsection{Partial-Sum Problem}

The one-dimensional partial-sum problem can be formally stated as follows. (The $d$-dimensional partial-sum problem will be defined in Section 7.) Let $A$ be an array of size $m$, indexed from 0 though $m-1$, whose value is known in advance. Let $M=\{0,1, \cdots, m-1\}$ be the set of index domain of $A$. Given a subset of $A^{\prime}$ s index domain $I \subset M$ at query time, we are interested in getting partial sum of $A$, specified by $I$ as:

$$
\operatorname{Psum}(A, I)=\sum_{i \in I} A[i] .
$$

EXAMPLE 1. For example, consider the following array $A$ with six elements:

$$
A=(259,401,680,937,452,63) \text {. }
$$

Let $I=\{0,1,5\}$, then $\operatorname{Psum}(A, I)=259+401+63=723$. Let $I=\{0,3,4\}$, then $\operatorname{Psum}(A, I)=259+937+452=1,648$.

We will use two metrics to measure the cost of solving the partial-sum problem: time overhead $T$ and space overhead $S$. The partial-sum computation requires an access to an element of $A$ followed by an addition of its value to an existing value (the cumulative partial sum). Thus, a time step can be modeled as the average time for accessing one array element and one arithmetic operation. We define $T$ of an algorithm as the maximum number of time steps 


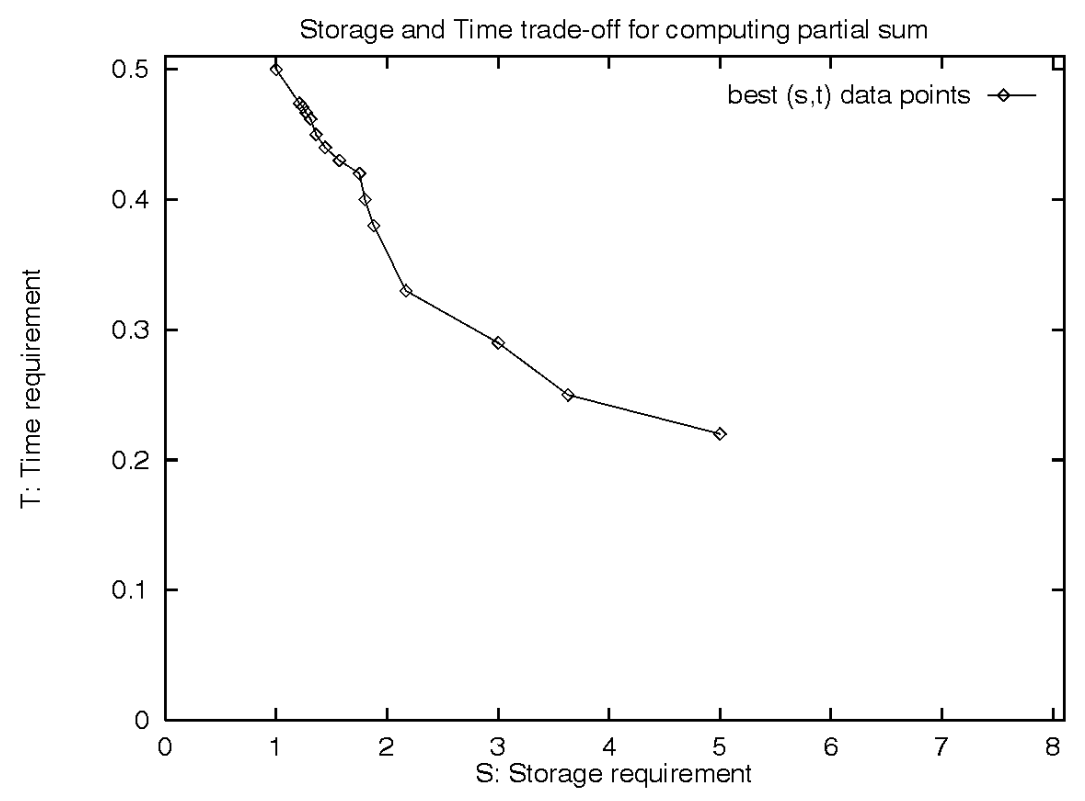

Fig. 1. The best $(s, t)$ data points for computing partial sum.

required by the algorithm (over all possible input $I$ ). We define $S$ as the number of storage cells required for the execution of the partial-sum operation. The storage may be used for the original array $A$ and for precomputed data that will help in achieving better response time. Clearly, a lower bound on $S$ is $m$ since at least the entire array $A$, or some encoded form of it, has to be stored. Without any precomputation, i.e., $S=m$, the worst-case time complexity is $T=m$ (which occurs when $I=M$ ). On the other hand, if one precomputes and stores all possible combinations of partial sums $\left(S=2^{m}-1\right)$, which is clearly infeasible for large $m$, only one data access is needed $(T=1)$.

A straightforward observation is that if we precompute only the total sum of $A$, say $A\left[{ }^{*}\right]=\sum_{i=0}^{m-1} A[i]$, then the worst-case time complexity for any partial sum can be reduced from $m$ to $\lceil m / 2\rceil$. This is because a partial sum can also be derived from $A\left[{ }^{*}\right]-\operatorname{Psum}\left(A, I^{\prime}\right)$ where $I^{\prime}=M-I$. For example, considering Example 1, we can store the sum of the elements $\left.A{ }^{*}\right]=2,792$. Assume $I=\{0,1,2,4,5\}$, then $\operatorname{Psum}(A, I)=A\left[^{*}\right]-A[3]=2,792-937=1,855$. We will consider the normalized measures for time and space. Namely, $s=S / m$ and $t=T / m$. Clearly, using the $A\left[^{*}\right]$ we can get $(s, t) \approx(1,0.5)$.

\subsection{Contributions}

The goal of the paper is to derive a suite of $(s, t)$ pairs, better than $(s, t) \approx(1,0.5)$. In particular, we will focus on finding $(s, t)$ for $t<0.5$ and $s$ being a small constant (say, less than five or so). The best $(s, t)$-pairs obtained in this paper are summarized in Fig. 1. (More detailed $(s, t)$ values are listed in Table 9 later.) For example, the entry $(s, t)=(1.44,0.44)$ implies that, with 44 percent additional storage, one can improve the query response time by about 12 percent (i.e., from $t=0.5$ to $t=0.44)$. Another entry $(s, t)=(2.17,0.33)$ means that if we roughly double the storage requirement, the query response time can be improved by about 34 percent.
The main contributions of the paper are as follows: First, we establish the connection between covering codes [12], [3] and the partial-sum problem. Second, we apply four known covering codes from [12], [5], and [4] to the partialsum problem to obtain algorithms with various space-time trade-offs. Third, we modify the requirements on covering codes to better reflect the partial-sum problem and devise new covering codes with respect to the new requirements. As a result, we further improve many of the $(s, t)$ points and give better space-time trade-offs.

Although we explicitly discuss only the SUM aggregation operation, the techniques presented apply to the other common OLAP aggregation operations of COUNT and AVERAGE-COUNT is a special case of SUM and AVERAGE can be obtained by keeping the 2-tuple (sum, count). In general, these techniques can be applied to any binary operation op for which there exists an inverse binary operation iop such that $a$ op $b$ iop $b=a$, for any $a$ and $b$ in the domain.

\subsection{Related Work}

Following the introduction of the data cube model in [9], there has been considerable research in developing algorithms for computing the data cube [1], for deciding what subset of a data cube to precompute [14], [11], [2], for estimating the size of multidimensional aggregates [19], and for indexing precomputed summaries [20], [15]. Related work also includes work done in the context of statistical databases [6] on indexing precomputed aggregates [21] and incrementally maintaining them [17]. Also relevant is the work on maintenance of materialized views [16] and processing of aggregation queries [8], [10], [22]. However, these works do not directly addresses efficient precomputation techniques for partial-sum queries.

Closest to the work presented in this paper is the accompanying paper [13], in which we consider range-sum queries over data cubes and give fast algorithms for them. A range-sum query obtains the sum over all selected cells of a 
data cube where the selection is specified by providing contiguous ranges of values for numeric dimensions. An example of a range-sum query over an insurance data cube is to find the revenue from customers with an age between 37 and 52, in a time from 1Qtr88 to 4Qtr96, in all of the U.S., and with auto insurance. Although a range-sum query can be viewed as a special case of the partial-sum query (thus, the general techniques proposed here can also be applied to the range-sum query), the techniques specialized for rangesum queries take advantage of the contiguous ranges of selection and should be preferred for better performance.

\subsection{Organization of the Paper}

The rest of the paper is organized as follows: In Section 2, we give a brief background on the covering codes that is pertinent to the partial-sum problem. In Section 3, we give main theorems that relate the properties of covering codes to the space and time complexities in solving the partialsum problem. In Section 4, we apply the known covering codes to the partial-sum problem. In Section 5, we modify the definition of the covering code by assuming all the weight- 1 vectors are included as codewords, in order to derive faster algorithms. In Section 6, we further modify the definition of the covering code based on a composition function. This results in further improvement in space and time overheads in solving the partial sum problem. Section 7 discusses partial-sum queries over multidimensional cubes. We conclude with a summary in Section 8.

\section{Covering Codes}

In this section, we briefly review some concepts from the theory of error-correcting codes [12], [3] that are pertinent to the partial-sum problem.

A code is a set of codewords where each codeword defines a valid string of digits. For the purposes of this paper, we are only interested in binary codes of fixed length. We will represent a binary vector in a bit string format and use the terms vector and bit string interchangeably depending on the context. The bit position of a length- $m$ bit string (or vector) is labeled from 0 through $m-1$ from left (the most significant bit) to right (the least significant bit). Also, $\mathcal{R}^{*}(V)$ denotes any bit-rotation of vector $V$ and " $\mid$ " denotes concatenation of two bit strings (vectors).

The Hamming weight of a length- $m$ binary vector $V=\left(b_{0} b_{1}\right.$ $\left.\cdots b_{m-1}\right)$ is $\sum_{i=0}^{m-1} b_{i}$, i.e., the number of 1-bits in this vector. The Hamming distance of two binary vectors $V$ and $V^{\prime}$, denoted Hamming $\left(V, V^{\prime}\right)$, is the Hamming weight of $V \otimes V^{\prime}$, where " $\otimes$ " is the bit-wise exclusive-or operator. For instance, the Hamming weight of the vector $V=(0010110)$ is three. The Hamming distance between $V=(0010110)$ and $V^{\prime}=(0010001)$ is three, which is the Hamming weight of $V \otimes V^{\prime}=(0000111)$. Throughout the paper, the weight of a codeword or a vector always means the Hamming weight.

The covering radius $R$ of a binary code is the maximal Hamming distance of any vector of the same length from a codeword (a vector in the code). A binary code $C$ is an $(m, K, R)$-covering code if
TABLE 1

THE $(5,7,1)$-COVERING CODE $\{(00000),(00111),(10000)$, (01000), (11011), (11101), (11110)\}

\begin{tabular}{|c|c|c|c|}
\hline weight & Vector & the closest codeword & dist. \\
\hline \hline 0 & $(00000)$ & itself & 0 \\
\hline 1 & $\mathcal{R}^{*}(00001)$ & $(00000)$ or itself & 0 or 1 \\
\hline \multirow{3}{*}{2} & $(00) \mid \mathcal{R}^{*}(011)$ & $(00111)$ & 1 \\
& $(01) \mid \mathcal{R}^{*}(001)$ & $(01000)$ & 1 \\
& $(10) \mid \mathcal{R}^{*}(001)$ & $(10000)$ & 1 \\
& $(11000)$ & $(01000)$ or $(10000)$ & 1 \\
\hline & $(00111)$ & itself & 0 \\
& $(01110)$ & $(11110)$ & 1 \\
& $(11100)$ & $(11110)$ or $(11101)$ & 1 \\
& $(11001)$ & $(11011)$ or $(11101)$ & 1 \\
3 & $(10011)$ & $(11011)$ & 1 \\
& $(01011)$ & $(11011)$ & 1 \\
& $(10110)$ & $(11110)$ & 1 \\
& $(01101)$ & $(11101)$ & 1 \\
& $(11010)$ & $(11011)$ or $(11110)$ & 1 \\
& $(10101)$ & $(11101)$ & 1 \\
\hline & $(01111)$ & $(00111)$ & 1 \\
& $(11110)$ & itself & 0 \\
& $(11101)$ & itself & 0 \\
& $(11011)$ & itself & 0 \\
& $(10111)$ & $(00111)$ & 1 \\
\hline 5 & $(11111)$ & any weight-4 codeword & 1 \\
\hline
\end{tabular}

$\mathcal{R}^{*}(V)$ denotes any bit-rotation of vector $V$ and " $\mid$ " denotes concatenation of two bit strings.

1) each codeword is of length $m$;

2) there are $K$ (legal) codewords in $C$ (out of all $2^{m}$ possible combinations in the vector space); and

3 ) the covering radius of the code is $R$.

EXAMPLE 2. The code $C=\{(00000),(11111)\}$ is a $(5,2,2)$ covering code because $m=5, K=2$, and $R=2$. For this code, $R=2$ because every binary vector of length five is within distance two from either (00000) or (11111). As another example, the code $C=\{(00000),(00111)$, (10000), (01000), (11011), (11101), (11110)\} can be verified from Table 1 as a $(5,7,1)$-covering code because all 32 vectors are within distance one from one of the seven codewords.

\section{Relating the Covering Radius of Codes to PARTIAL SUMS}

\subsection{A Motivating Example}

We first give a motivating example based on the $(5,7,1)$ covering code. Suppose the array $A$ is of size $m=5$ and the initial values of $A[0]$ through $A[4]$ are known. We first precompute the partial sums corresponding to all seven codewords of the $(5,7,1)$-covering code. For instance, corresponding to the codeword (00111), the precomputed partial sum is $A[2]+A[3]+A[4]$. Note that the corresponding partial sum for (00000) is zero and need not be computed. Also, the corresponding partial sums for (10000) and (01000) are already known as part of the original array elements. 
Now suppose the partial sum query is $\operatorname{Psum}(A, I)$, where $I=\{0,2,3,4\}$, i.e., corresponding to the vector (10111). We can derive its partial sum as the sum of the precomputed partial sum corresponding to codeword (00111) and the value of $A[0]$. In fact, any partial sum $\operatorname{Psum}(A, I)$ for this example can be derived as some precomputed partial sum plus or minus some array value. This is because the radius of the $(5,7,1)$-covering code is 1 . We are now ready to relate covering codes to the partial-sum problem formally.

\subsection{Using Covering Codes to Solve Partial Sums}

Given a length- $m$ covering code $C$ and any $m$-bit vector $V$, we use $f_{t}(m)$ and $f_{s}(m)$ to denote the time and associated space overheads, respectively, in deriving the index to codeword in $C$ that is closest to $V$. Note that $f_{t}(m)$ and $f_{s}(m)$ may depend on certain property of the code, in addition to the length of the codeword. However, for notational simplicity, we omit the parameter $C$ in $f_{t}$ and $f_{s}$.

For convenience, we define an $m$-bit mask of $I$ as mask (I) $=\left(b_{0} b_{1} \cdots b_{m-1}\right)$ where $b_{i}=1$ if $i \in I$, and $b_{i}=0$ otherwise. Also, if $V=$ mask $(I)$, then the set $I$ will be called the support of vector $V$, denoted $\operatorname{support}(V)=I$. (Support and mask are inverse functions). For instance, if $m=5, I=\{0,1,3\}$, then $\operatorname{mask}(I)=(11010)$. Also, $\operatorname{support}((11010))=\{0,1,3\}$.

LEMMA 1. Given an $(m, K, R)$-covering code with $c$ codewords of Hamming weight 1 or 0 in the code, we can construct an algorithm to derive the partial sum $\operatorname{Psum}(A, I)$ in time $T=$ $R+f_{t}(m)+1$ and in space $S=m+K-c+f_{s}(m)$.

PROOF. Denote the $K$ codewords (vectors) by $V_{1}, V_{2}, \cdots, V_{K}$. Let $I_{i}=\operatorname{support}\left(V_{i}\right)$. Without loss of generality, assume that the $c$ codewords with weight 1 or 0 are the first $c$ on the list. (Thus, the partial sum for each of $I_{1}, I_{2}, \cdots$, $I_{c}$ is already known as they correspond to entries in array A.) We will precompute and store the partial sums for $K-c$ different subsets specified by $I_{c+1}, I_{c+2}$, $\cdots, I_{K}$, respectively. This requires a space overhead of $K-c$. Given an index subset parameter $I$ at run time, let $V=\operatorname{mask}(I)$. We first find an index $i$ such that $V_{i}$ is the closest codeword from $V$. This requires a time overhead of $f_{t}(m)$ and a space overhead of $f_{s}(m)$. Then, we access the precomputed $\operatorname{Psum}\left(A, I_{i}\right)$ in one step. Since $V$ is at most distance $R$ away from $V_{i}$ (due to the property of an $(m, K, R)$-covering code), the partial sum $\operatorname{Psum}(A, I)$ can be obtained from $\operatorname{Psum}\left(A, I_{i}\right)$ by accessing and adding or subtracting up to $R$ elements of $A$, which correspond to the 1-bit positions of $V \otimes V_{i}$. Thus, the time overhead for this modification is at most $R$. Overall, we have $T=R+f_{t}(m)+1$ and $S=m+$ $K-c+f_{s}(m)$.

\subsection{Reducing Space Overhead}

Recall that array $A$ is of size $m$. The above lemma applies any covering code of length $m$ to the entire array. However, many covering codes have small $R$ and large $K$ relative to $m$ [12], [5], [4]. Applying these covering codes directly to the entire array typically yields an unreasonable space overhead, even though the time is much improved. Furthermore, the space overhead depends on the array size $m$. In the following theorem, we will partition the array into blocks of size $n$ and apply length- $n$ covering codes to each block.
THEOREM 2. Given an $(n, K, R)$-covering code with $c$ codewords of Hamming weight 1 or 0 in the code, we can construct an algorithm to derive the partial sum Psum $(A, I)$ in time

$$
T \approx\left(R+f_{t}(n)+1\right) \frac{m}{n}
$$

and in space

$$
S \approx(n+K-c) \frac{m}{n}+f_{s}(n) .
$$

PROOF. Assume first that $m$ is a multiple of $n$. Logically partition the array $A$ into $m / n$ blocks of size $n$ each. Let $x=$ $m / n$. Denote them as $A_{0}, \cdots, A_{x-1}$. Also partition $I$ into $I_{0}, \cdots, I_{x-1}$. Then, $\operatorname{Psum}(A, I)=\sum_{i=0}^{x-1} \operatorname{Psum}\left(A_{i}, I_{i}\right)$. To derive $\operatorname{Psum}\left(A_{i}, I_{i}\right)$ for each $0 \leq i<x$, we apply the algorithm constructed in Lemma 1, which incurs overhead $T_{i}=R+f_{t}(n)+1$ in time and $S_{i}=n+K-c+f_{s}(n)$ in space. The space overhead $f_{s}(n)$ is the same for all $i$ because the same covering code is applied. Thus, the overall time complexity is $T=\sum_{i=0}^{x-1} T_{i}=\left(R+f_{t}(n)+1\right) \frac{m}{n}$ and the overall space overhead is

$S=\left(\sum_{i=0}^{x-1}\left(S_{i}-f_{s}(n)\right)\right)+f_{s}(n)=(n+K-c) \frac{m}{n}+f_{s}(n)$.

When $m$ is not a multiple of $n$, we can extend the array $A$ to a size $m^{\prime}=\lceil m / n\rceil n$ by padding $m^{\prime}-m$ elements of value 0 . This introduces the approximation sign in the complexities of $T$ and $S$.

By comparing the time and space complexities of this theorem to that of Lemma 1, it may appear that both time and space complexities are worse in this theorem. Note, however, that $R$ is a function of the vector length ( $m$ or $n)$ for a fixed $K$.

\subsection{Implementation Using Look-up Tables}

In this subsection, we give a concrete example of implementation based on Theorem 2 and give a general estimate of the time and space overhead $\left(f_{t}(n)\right.$ and $\left.f_{s}(n)\right)$ through the use of look-up tables.

We assume $m$ is a multiple of $n$. (If not, we can extend the size of $A$ to $\lceil\mathrm{m} / n\rceil n$ by padding zero elements to $A$.) First, we will restructure $A$ as a two-dimensional array $A[i, j]$, where $i$ indexes a block, $0 \leq i<\lceil m / n\rceil$, and $j$ indexes an element of $A$ within the block, $0 \leq j<n$. Thus, the new $A[i, j]$ is the same as the old $A[n i+j]$. Then, for each block $i$, we precompute the $K-c$ partial sums and store their value in $A[i, j]$ for $n \leq j<n+K-c$ in some arbitrary order (though the order is the same for all blocks).

The augmented two-dimensional array $A$ is a partial-sum look-up table including the original elements of $A$ (i.e., all $n$ codewords with a Hamming weight 1 for each block) and selected precomputed partial sums for each block of $A$. Table 2 shows an example of the partial-sum look-up table for the $i$ th block of $A$, based on the $(5,7,1)$-covering code described in Table 1 . The codewords of the $(5,7,1)$-covering code are marked with "** in the table. Also note that codeword (00000) is not needed in the table because the corresponding 
TABLE 2

THE PARTIAL-SUM LOOK-UP TABLE FOR THE $i$ TH BLOCK OF $A$ BASED ON THE $(5,7,1)$-COVERING CODE

\begin{tabular}{|c|c|c|}
\hline & Vector & Initial or precomputed value \\
\hline \hline$[i, 0]$ & $(10000)^{*}$ & $A[5 i]$ \\
\hline$[i, 1]$ & $(01000)^{*}$ & $A[5 i+1]$ \\
\hline$[i, 2]$ & $(00100)$ & $A[5 i+2]$ \\
\hline$[i, 3]$ & $(00010)$ & $A[5 i+3]$ \\
\hline$[i, 4]$ & $(00001)$ & $A[5 i+4]$ \\
\hline \hline$[i, 5]$ & $(00111)^{*}$ & $A[5 i+2]+A[5 i+3]+A[5 i+4]$ \\
\hline$[i, 6]$ & $(11011)^{*}$ & $A[5 i]+A[5 i+1]+A[5 i+3]+A[5 i+4]$ \\
\hline$[i, 7]$ & $(11101)^{*}$ & $A[5 i]+A[5 i+1]+A[5 i+2]+A[5 i+4]$ \\
\hline$[i, 8]$ & $(11110)^{*}$ & $A[5 i]+A[5 i+1]+A[5 i+2]+A[5 i+3]$ \\
\hline
\end{tabular}

The codewords of the $(5,7,1)$-covering code are marked with "*". Also, (00000) is not needed.

partial-sum is 0 , which can be omitted. The second column in the table is included for clarity only and is not needed in the look-up table. There are $\lceil m / n\rceil$ such tables, one for each block and each of size $n+K-c$. Thus, a total of size $(n+K-$ c) $\lceil\mathrm{m} / \mathrm{n}\rceil$ is needed for the partial- look-up table.

Second, we will create an index look-up table with $2^{n}-1$ entries, indexed from 1 to $2^{n}-1$. For each entry, we store a list of (index, sign)-pairs, denoted $\left(j_{1}, s_{1}\right),\left(j_{2}, s_{2}\right), \cdots$, so that the partial sum of the $i$ th block with vector $V$ can be derived as $\sum\left(s_{x} * A\left[i, j_{x}\right]\right)$ for all $\left(j_{x}, s_{x}\right)$-pairs defined in the list. Note that the list has at most $R+1$ pairs. Following the same example, Table 3 gives an example of the index lookup table. In the table, an index of " -1 " marks the end of the list and a question mark "?" implies a don't-care value. As before, the "vector-column" is included here for clarity only and is not needed in the look-up table. Also, it is possible to build the table so that the sign for the first index is always positive (such as the example given) and can be omitted.

As an example, assume the $i$ th block of $I$ is (00011). We use the value of (00011), which is three, to index this table. According to the table, the partial sum corresponding to (00011) in the $i$-block can be derived by $A[i, 3]+\mathrm{A}[i, 4]$. Then, from Table $2, A[i, 3]$ and $A[i, 4]$ are prestored with values $A[5 i+3]$ and $A[5 i+4]$, respectively. As another example, assume the $i$ th block of $I$ is (01011). According to Table 3 , the partial sum is $A[i, 6]-A[i, 0]$, which, according to Table 2 , yields $(A[5 i]+A[5 i+1]+A[5 i+3]+A[5 i+4])-$ $A[5 i]=A[5 i+1]+A[5 i+3]+A[5 i+4]$. The size of the index look-up table is bounded by $f_{s}(n)=O\left(2^{n} R\right)$ from above.

With the implementation of the index look-up table, the time overhead for finding the closest codeword of an $n$-bit vector, $f_{t}(n)$, becomes the time to index an array of $2^{n}$ entries. Since the same covering code is used for all blocks, the same index look-up table will be used for indexing for all blocks.

\section{Applying Known Covering Codes}

In this section, we will apply some known covering codes to the partial-sum problem, based on Theorem 2. Different covering codes lead to different look-up tables and, hence, different space-time trade-offs. We have chosen $(n, K, R)$ -
TABLE 3

THE INDEX LOOK-UP TABLE

\begin{tabular}{|c|c|c|c|c|c|}
\hline Index & Vector & 1st index & 1st sign & 2nd index & 2nd sign \\
\hline \hline 1 & $(00001)$ & 4 & +1 & -1 & $?$ \\
\hline 2 & $(00010)$ & 3 & +1 & -1 & $?$ \\
\hline 3 & $(00011)$ & 3 & +1 & 4 & +1 \\
\hline 4 & $(00100)$ & 2 & +1 & -1 & $?$ \\
\hline 5 & $(00101)$ & 2 & +1 & 4 & +1 \\
\hline 6 & $(00110)$ & 2 & +1 & 3 & +1 \\
\hline 7 & $(00111)$ & 5 & +1 & -1 & $?$ \\
\hline 8 & $(01000)$ & 1 & +1 & -1 & $?$ \\
\hline 9 & $(01001)$ & 1 & +1 & 4 & +1 \\
\hline 10 & $(01010)$ & 1 & +1 & 3 & +1 \\
\hline 11 & $(01011)$ & 6 & +1 & 0 & -1 \\
\hline 12 & $(01100)$ & 1 & +1 & 2 & +1 \\
\hline 13 & $(01101)$ & 7 & +1 & 0 & -1 \\
\hline 14 & $(01110)$ & 8 & +1 & 0 & -1 \\
\hline 15 & $(01111)$ & 5 & +1 & 1 & +1 \\
\hline 16 & $(10000)$ & 0 & +1 & -1 & $?$ \\
\hline 17 & $(10001)$ & 0 & +1 & 4 & +1 \\
\hline 18 & $(10010)$ & 0 & +1 & 3 & +1 \\
\hline 19 & $(10011)$ & 6 & +1 & 1 & -1 \\
\hline 20 & $(10100)$ & 0 & +1 & 2 & +1 \\
\hline 21 & $(10101)$ & 7 & +1 & 1 & -1 \\
\hline 22 & $(10110)$ & 8 & +1 & 1 & -1 \\
\hline 23 & $(10111)$ & 5 & +1 & 0 & +1 \\
\hline 24 & $(11000)$ & 0 & +1 & 1 & +1 \\
\hline 25 & $(11001)$ & 7 & +1 & 2 & -1 \\
\hline 26 & $(11010)$ & 8 & +1 & 2 & -1 \\
\hline 27 & $(11011)$ & 6 & +1 & -1 & $?$ \\
\hline 28 & $(11100)$ & 8 & +1 & 3 & -1 \\
\hline 29 & $(11101)$ & 7 & +1 & -1 & $?$ \\
\hline 30 & $(11110)$ & 8 & +1 & -1 & $?$ \\
\hline 31 & $(11111)$ & 8 & +1 & 4 & +1 \\
\hline
\end{tabular}

covering codes with combinations of minimum radius $R$ and minimum number of codewords $K$, given the length of codewords $n$. Specifically, we consider four classes of codes: two classes for two different generalizations of Hamming code $(7,16,1)$, one class for the generalization of $(5,7,1)$ code, and one class for the generalization of $(6,12,1)$ code. These are the only codes that yielded useful $(s, t)$-pairs among all the codes included in [12], [5], and [4].

\subsection{The $(7+2 i, 16, i+1)$-Covering Codes}

It was shown in $[12]$ that the $(7,16,1)$ Hamming code can be generalized to $(7+2 i, 16, i+1)$-covering codes, for all $i \geq 0$. For example, $(9,16,2)$ and $(11,16,3)$ are in this family of codes.

\subsection{The $\left(n+i, 2^{i} K, R\right)$-Covering Codes}

An $(n, K, R)$-covering code can also be extended to an $(n+i$, $\left.2^{i} K, R\right)$-covering code simply by replicating the same set of codewords $2^{i}$ times, each in a copy of the $2^{n}$ vectors. Thus, $(7,16,1)$ Hamming code also generalizes to $\left(7+i, 2^{i+4}, 1\right)$ covering codes for all $i \geq 0$. However, for many $n \geq 9$, better $(n, K, 1)$-covering codes than the naive extension from $(7,16,1)$ are known [5], [4]. In particular, $(9,62,1)$ is such a code included in [4].

\subsection{Piecewise Constant Codes}

A family of codes, called piecewise constant codes, was introduced in [5]. We include its definition and give an example here for easy reading. 
TABLE 4

A $(5,7,1)$ Piecewise Constant Code AS A COVERING CODE

\begin{tabular}{|l|l|}
\hline 00 & 000 \\
\hline 00 & 111 \\
\hline 10 & 000 \\
01 & 000 \\
\hline 11 & 011 \\
11 & 101 \\
11 & 110 \\
\hline
\end{tabular}

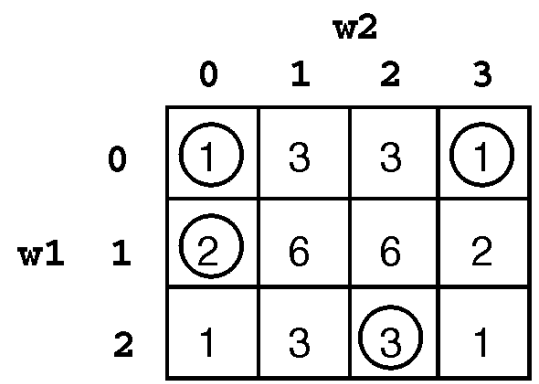

Fig. 2. Two-dimensional array representing the $(5,7,1)$ covering code of Table 4.

First, the length $n$ of a codeword is partitioned into $t$ parts: $n=n_{1}+n_{2}+\cdots+n_{t}$. Each codeword $c$ is partitioned in the same way, as

$$
c=\left(c^{(1)}, c^{(2)}, \cdots, c^{(t)}\right),
$$

where length $\left(c^{(i)}\right)=n_{i}$. Then, $C$ is a piecewise constant code if it has the property that "if $C$ contains one word with weights

$$
w t\left(c^{(1)}\right)=w_{1}, \cdots, w t\left(c^{(t)}\right)=w_{t},
$$

then it contains all such words."

For example, Table 4 shows a piecewise constant code of length $n=5$ corresponding to the partition $n=n_{1}+n_{2}$, where $n_{1}=2$ and $n_{2}=3$. There are seven codewords, corresponding to the weights

$$
\begin{array}{ll}
w_{1}=0, w_{2}=0, & 1 \text { word, } \\
w_{1}=0, w_{2}=3, & 1 \text { word, } \\
w_{1}=1, w_{2}=0, & 2 \text { words, } \\
w_{1}=2, w_{2}=2, & 3 \text { words. }
\end{array}
$$

Any piecewise constant code of length five partitioned as $5=n_{1}+n_{2}=2+3$ can be represented by a subset of the two-dimensional array of cells shown in Fig. 2. The cell at position $\left(w_{1}, w_{2}\right)$ represents the set of vectors $c=\left(c^{(1)}, c^{(2)}\right)$ with $w t\left(c^{(1)}\right)=w_{1}, w t\left(c^{(2)}\right)=w_{2}$. There are

$$
\left(\begin{array}{c}
n_{1} \\
w_{1}
\end{array}\right)\left(\begin{array}{c}
n_{2} \\
w_{2}
\end{array}\right)=\left(\begin{array}{c}
2 \\
w_{1}
\end{array}\right)\left(\begin{array}{c}
3 \\
w_{2}
\end{array}\right)
$$

such vectors, and this number is written in the cell. A piecewise constant code is then specified by circling some of the cells in the array, and the number of codewords is the
TABLE 5

A $(6,12,1)$ PIECEWISE Constant Code AS A COVERING CODE

\begin{tabular}{|l|l|}
\hline 000 & 100 \\
000 & 010 \\
000 & 001 \\
\hline 100 & 111 \\
010 & 111 \\
001 & 111 \\
\hline 011 & 000 \\
101 & 000 \\
110 & 000 \\
\hline 111 & 011 \\
111 & 101 \\
111 & 110 \\
\hline
\end{tabular}

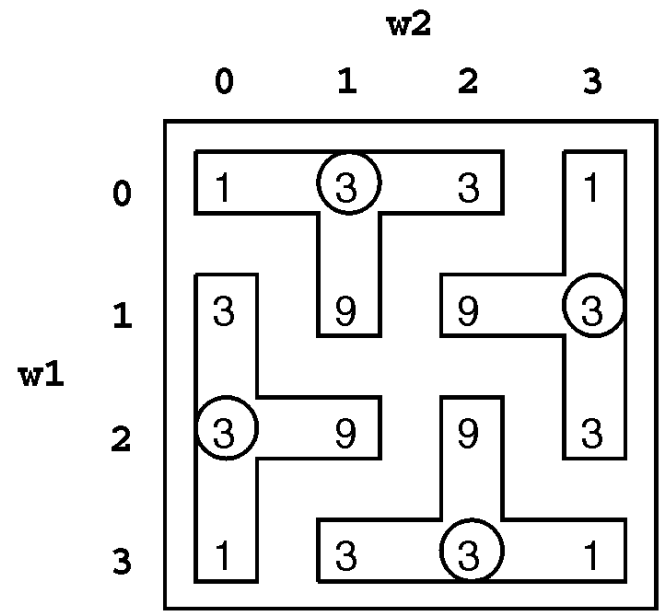

Fig. 3. Two-dimensional array representing the $(6,12,1)$ covering code of Table 5 and showing the Manhattan "spheres" of covering radius 1 around the circled cells.

sum of the circled numbers. The four circled cells in Fig. 2 represent the code of Table 4 , and there are a total of seven codewords.

Piecewise constant codes have the desirable property that the covering radius $R$ is easy to calculate from this array of cells. This is because radius $R$ is simply the maximal distance of any cell from the code (i.e., from the nearest circled cell), when the distance between two cells is measured in the Manhattan metric. In Fig. 2, the Manhattan distance between two cells is the number of horizontal and vertical steps needed to move from one to the other. It is clear that, in Fig. 2, every cell is within Manhattan distance 1 of a circled cell, so the covering radius $R$ is 1 . Thus, we have an $(n, K, R)=(5,7,1)$ covering code.

A second example of a piecewise constant code is given in Table 5 and Fig. 3. This corresponds to the partition $6=3+3$ and contains 12 codewords. Fig. 3 shows the "spheres" of Manhattan radius 1 around the codewords, proving that $R=1$. Thus, we have an $(n, K, R)=(6,12,1)$ covering code. 


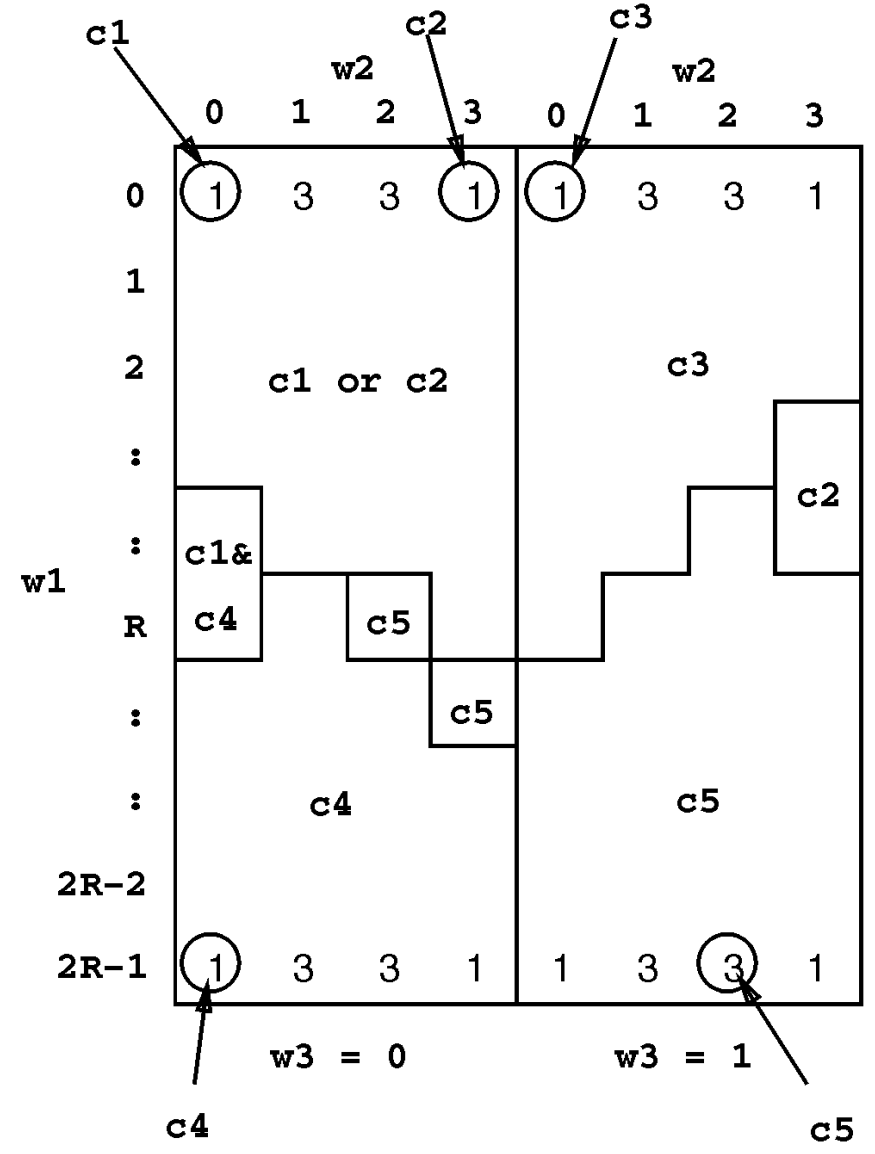

Fig. 4. Three-dimensional array showing a family of piecewise constant codes as the $(2 R+3,7, R)$-covering codes.

\subsection{The $(2 R+3,7, R)$-Covering Codes}

Fig. 4 shows a family of piecewise constant codes, given in [5], which are $(2 R+3,7, R)$-covering codes. The code is partitioned into three parts: $n=(2 R-1)+3+1=2 R+3$. The figure shows certain key boundaries of the Manhattan spheres of radius $R$. Each region is marked by the codeword(s) covering it. Recall that the number of codewords, seven, is the sum of the circled numbers. In fact, the family of $(2 R+3,7, R)$-covering codes can be viewed as a generalization of the $(5,7,1)$ code (Table 4$)$ through an amalgamated direct sum technique described in [12] and [5].

\subsection{The $(2 R+4,12, R)$-Covering Codes}

Fig. 5 shows another family of piecewise constant codes, which are $(2 R+4,12, R)$-covering codes. The code is partitioned into three parts: $n=(2 R-2)+3+3=2 R+4$. As before, the figure shows certain key boundaries of the Manhattan spheres of radius $R$ and each region is marked by the codeword(s) covering it. Formally, the family of $(2 R+4,12, R)$ covering codes can be viewed as a result of applying the amalgamated direct sum of $(6,12,1)$ code with $(3,2,1)$ code iteratively [12], [5].

\subsection{Results}

The results of applying the above codes to the partial-sum problem are summarized in Table 6 . The results show a spectrum of space-time trade-offs and one can choose an operating point depending upon the objective. Recall that we defined the total space required, including the original array of size $m$, as sm. (That is, $s-1$ is the multiplicative overhead.) There is, however, an additive overhead of $f_{s}(n)$ $=O\left(2^{n} R\right)$ not included in this and subsequent tables with an s-column.

\section{Single-Weight-EXTENDEd Covering Codes}

In this section, we will modify the property of covering codes to better reflect the partial-sum problem. We will first define a new type of covering codes, which we shall call the single-weight-extended covering codes. Then, we present a general theorem relating this type of covering codes to the partial-sum problem. Finally, we will devise a class of covering codes of this type.

\subsection{Specialized Covering Codes for Partial Sums}

In applying existing $(n, K, R)$-covering codes to the partialsum problem in the previous section, we chose codes with combinations of minimum radius $R$ and minimum number of codewords $K$, given the length of codewords $n$. Minimizing the time for the partial-sum problem is different from minimizing the covering radius $R$ given length $n$ and $K$ codewords of an $(n, K, R)$-covering code in two ways. First, the all- 0 vector $(00 \cdots 0)$ need not be covered (since the corresponding partial sum is always 0 ). Second, the $n$ weight 1 vectors can be included in the covering code without space cost since they are present in array $A$, which may reduce $R$.

We, therefore, define the single-weight-extended covering code. To derive efficient algorithms for partial sums, our new objective is to derive $\left(n, K^{\prime}, R\right)^{+}$-covering codes with combinations of minimum $R$ and $K^{\prime}$, for various given small $n$.

DEFINITION 1. A binary code $C$ is an $\left(n, K^{\prime}, R\right)$ single-weightextended covering code, denoted $\left(n, K^{\prime}, R\right)^{+}$-covering code, if

1) each codeword is of length $n$;

2) there are $K^{\prime}$ codewords in $C$; and

3) letting $C^{\prime}=C \cup\left\{\mathcal{R}^{*}(00 \cdots 01)\right\}$, i.e., $C$ extended with all $n$ weight-1 vectors, the covering radius of the code $C^{\prime}$ is $R$.

Since the all- 0 vector is always distance one from any weight- 1 vector and $R \geq 1$ for all our cases, covering the all- 0 vector (to be consistent with the definition of covering codes) does not increase the complexities of $K^{\prime}$ and $R$ of the code. Clearly, an $(n, K, R)$-covering code is also an $(n, K-c, R)^{+}$covering code. We will use $K^{\prime}$ throughout this section to denote the number of codewords excluding the all-0 vector and all weight- 1 vectors.

THEOREM 3. Given an $\left(n, K^{\prime}, R\right)^{+}$-covering code, we can construct an algorithm to derive the partial sum $\operatorname{Psum}(A, I)$ in time $T \approx\left(R+f_{t}(n)+1\right) \frac{m}{n}$ and in space $S \approx\left(n+K^{\prime}\right) \frac{m}{n}+f_{s}(n)$.

PROOF. Follows from Theorem 2 and Definition 1.

\subsection{The $(2 R+3,4, R)^{+}$-Covering Codes}

We now give a construction of a $(2 R+3,4, R)^{+}$-covering code $C$ for all $R \geq 1$ and prove its correctness. The construction can 


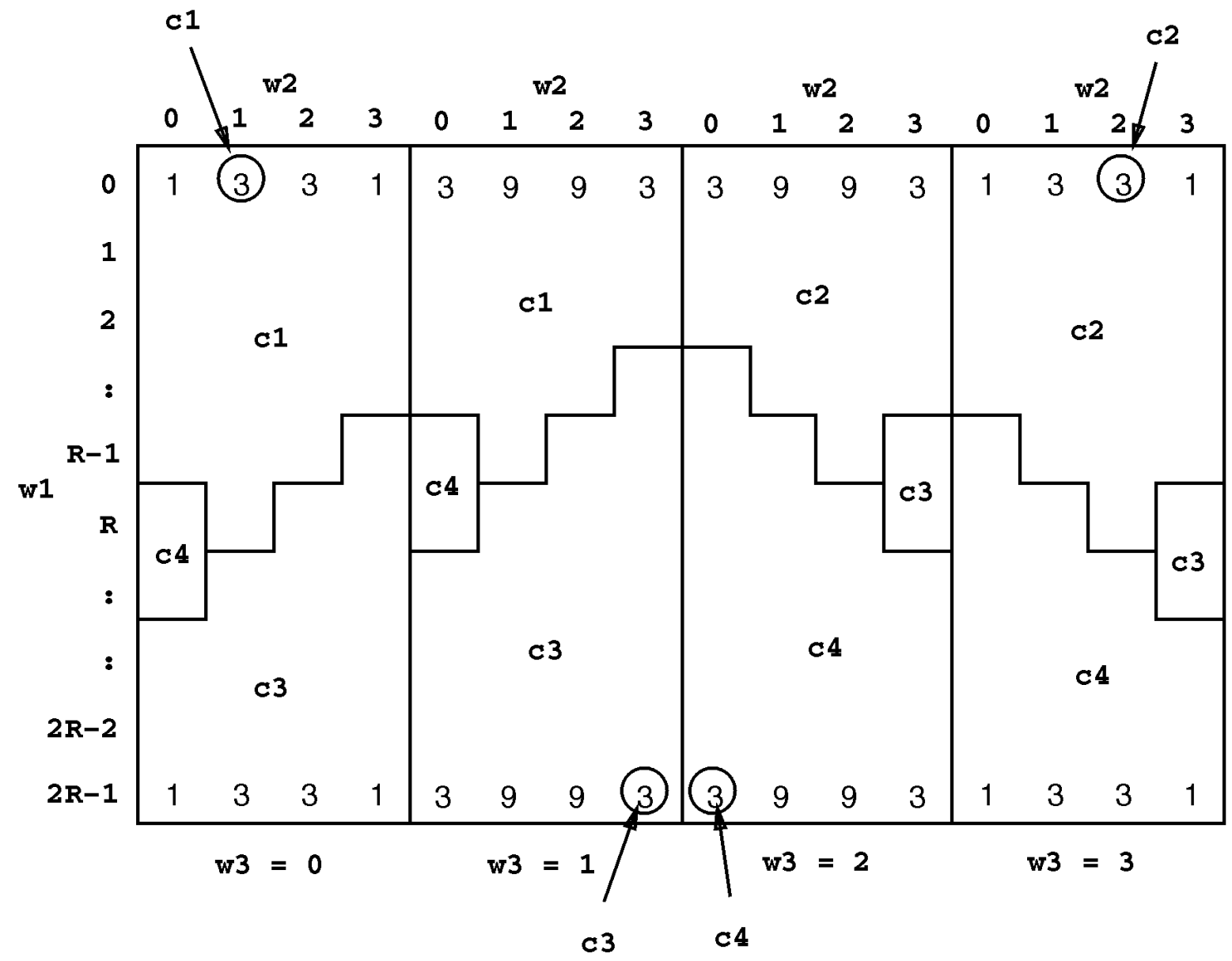

Fig. 5. Three-dimensional array showing a family of piecewise constant codes as the $(2 R+4,12, R)$-covering codes.

be defined by Fig. 6, which is modified from Fig. 4 by taking into account that all weight- 1 codewords will be included. In Fig. 6, the $2 R+3$ weight- 1 codewords are represented by the three dashed circles $((2 R-1)+3+1=2 R+3)$, and denoted by $c_{5}, c_{6}$, and $c_{7}$. The $K^{\prime}=4$ codewords are denoted as $c_{1}, \cdots, c_{4}$, respectively. As before, each region is marked by the codeword(s) covering it.

We now give a formal definition and proof of a $(2 R+3$, $4, R)^{+}$-covering code for any positive integer $R$. Recall that each codeword has $2 R+3$ bits. We will use $Y$ to denote the all-1 vector $(11 \cdots 1)$ of length $2 R-1$ and use $Z$ to denote the all- 0 vector $(00 \cdots 0)$ of length $2 R-1$. Then, the four codewords in the $(2 R+3,4, R)^{+}$-covering code, consistent with Fig. 6, can be denoted as

$$
\begin{gathered}
C=\left\{c_{1}=(Z \mid 1111), c_{2}=(Y \mid 1111),\right. \\
\left.c_{3}=(Y \mid 1110), c_{4}=(Y \mid 0001)\right\} .
\end{gathered}
$$

THEOREM 4. The code $C$ defined above is a $(2 R+3,4, R)^{+}$covering code.

ProOF. Consider any vector $V$ of length $2 R+3$. Partition the vector $V$ into three subvectors, from left to right: $V_{1}$ of length $2 R-1, V_{2}$ of length three, and $V_{3}$ of length one. Let $w_{1}, w_{2}$, and $w_{3}$ be the Hamming weight of $V_{1}, V_{2}$, and $V_{3}$, respectively. Let $W$ be the set of all length- $(2 R+3)$ weight- 1 vectors, i.e., $W$ includes $c_{5}, c_{6}, c_{7}$ of the figure.
Recall from Definition 1 that the covering radius of a single-weight-extended covering code is defined with respect to $C \cup W$. Consider the following three cases that cover all combinations of $V$ :

Case 1: $w_{3}=0$. If $w_{1}+w_{2} \geq R+2$ (the lower left region of the figure) then the Hamming distance of $V$ and $c_{3}=$ $(Y \mid 1110)$ is at most $(2 R+2)-(R+2)=R$. Otherwise (the upper left region), $w_{1}+w_{2} \leq R+1$ and there exists a vector in $W$ whose Hamming distance is at most $R$ from $V$.

Case 2: $w_{3}=1$ and $w_{1} \leq R-1$ (the upper right region). If $w_{2} \leq 1$, then the Hamming distance between $V$ and $c_{7}=$ $(Z \mid 0001) \in W$ is $\operatorname{Hamming}\left(V_{1}, Z\right)+w_{2}=w_{1}+w_{2} \leq(R-1)$ $+1=R$. Otherwise, $w_{2} \geq 2$ and the Hamming distance between $V$ and $c_{1}=(Z \mid 1111)$ is Hamming $\left(V_{1}, Z\right)+\left(3-w_{2}\right)$ $\leq(R-1)+1=R$.

Case 3: $w_{3}=1$ and $w_{1} \geq R$ (the lower right region). If $w_{2} \leq 1$, then the Hamming distance between $V$ and $c_{4}=$ $(Y \mid 0001)$ is $\operatorname{Hamming}\left(V_{1}, Y\right)+w_{2} \leq((2 R-1)-R)+1=R$. Otherwise, $w_{2} \geq 2$ and the Hamming distance between $V$ and $c_{2}=(Y \mid 1111)$ is Hamming $\left(V_{1}, Y\right)+\left(3-w_{2}\right) \leq$ $((2 R-1)-R)+1=R$. 


\subsection{Results}

Table 7 summarizes the best $(s, t)$-pairs obtained based on the previous Table 6 and the class of new codes devised in this section. Note that the $(14,12,5)$-covering code from Table 6 is removed from the new table because the new $(7,4,2)^{+}$-covering code has a better $(s, t)$-pair.

\section{FURTHER IMPROVEMENTS}

We now further modify the definition of the covering code by adding a composition function, resulting in a new class of codes, which we shall call composition-extended covering codes. The main result (space and time overheads) for the partial-sum problem implied by the new class of covering codes is described in Theorem 6 . The key to the new class of codes is that a partial sum may be written by a sum or difference of two other partial sums. Thus, some efficient coding scheme can be implemented using this.

\subsection{Covering Codes with Composition Function}

Let $\ominus$ be the bit-wise or operator, $\oplus$ the bit-wise and operator, and $\otimes$ the bit-wise exclusive-or operator. Let $\perp$ denote an undefined value.

DEFINITION 2. Define a composition function of two binary vectors $V$ and $V^{\prime}$ as follows:

$$
\operatorname{comp}\left(V, V^{\prime}\right)=V \odot V^{\prime}= \begin{cases}V \ominus V^{\prime}, & \text { if } V \oplus V^{\prime}=0 ; \\ V \otimes V^{\prime}, & \text { if } V \oplus V^{\prime}=V \\ & \text { or } V \oplus V^{\prime}=V^{\prime} ; \\ \perp, & \text { otherwise. }\end{cases}
$$

For example, $\operatorname{comp}((001),(011))=(010), \operatorname{comp}((001),(010))$ $=(011))$, and $\operatorname{comp}((011),(110))=\perp$. The intuition behind this function lies in the following lemma:

LEMMA 5. Let $V, V^{\prime}$ be two n-bit vectors where $V^{\prime \prime}=\operatorname{comp}\left(V, V^{\prime}\right)$ $\neq \perp$. Also let $I, I^{\prime}$, and $I^{\prime \prime}$ be support $(V)$, support $\left(V^{\prime}\right)$, and $\operatorname{support}\left(V^{\prime \prime}\right)$, respectively. Then, given $\operatorname{Psum}(A, I)$ and $\operatorname{Psum}\left(A, I^{\prime}\right)$, one can derive Psum $\left(A, I^{\prime \prime}\right)$ in one addition or subtraction operation.

ProOF. By Definition 2, it can be shown that

$$
\operatorname{Psum}\left(A, I^{\prime \prime}\right)= \begin{cases}\operatorname{Psum}(A, I)+\operatorname{Psum}\left(A, I^{\prime}\right), & \text { if } V \oplus V^{\prime}=0 ; \\ \operatorname{Psum}\left(A, I^{\prime}\right)-\operatorname{Psum}(A, I), & \text { if } V \oplus V^{\prime}=V ; \\ \operatorname{Psum}(A, I)-P \operatorname{sum}\left(A, I^{\prime}\right), & \text { if } V \oplus V^{\prime}=V^{\prime} .\end{cases}
$$

For consistency, we will let $\operatorname{comp}\left(V, V^{\prime}\right)=\perp$ if either $V=\perp$ or $V^{\prime}=\perp$. (All other rules still follow Definition 2.) We assume $\odot$ operator associates from left to right, i.e., $V \odot V^{\prime} \odot V^{\prime \prime}$ $=\left(V \odot V^{\prime}\right) \odot V^{\prime \prime}$. Note that $\odot$ is commutative, but not associative. For instance, $(1100) \odot(1101) \odot(1010)=(1011)$, while $(1100) \odot((1101) \odot(1010))=\perp$.

DEFINITION 3. A binary code $C$ is an $\left(n, K^{\prime \prime}, R\right)$ compositionextended covering code, denoted $\left(n, K^{\prime \prime}, R\right)^{*}$-covering code, if

1) each codeword is of length $n$,

2) there are $K^{\prime \prime}$ codewords in $C$, and

3) every length-n noncodeword vector $V \notin C$ can be derived by up to $R$ compositions of $R+1$ codewords, i.e.,
TABLE 6

BEST CHOICES OF SAND $T$ BASED ON EXISTING COVERING CODES

\begin{tabular}{|c||c|c|c|c|c|c|}
\hline$n$ & $K$ & $R$ & $c$ & $s$ & $t$ & ref. \\
\hline \hline$m$ & 2 & $m / 2$ & 1 & $1+1 / m$ & 0.50 & \\
odd $n$ & 7 & $(n-3) / 2$ & 2 & $1+5 / n$ & $0.5-\frac{1}{2 n}$ & $\S 4.4$ \\
19 & 7 & 8 & 2 & 1.26 & 0.474 & $\S 4.4$ \\
17 & 7 & 7 & 2 & 1.29 & 0.471 & $\S 4.4$ \\
15 & 7 & 6 & 2 & 1.33 & 0.467 & $\S 4.4$ \\
13 & 7 & 5 & 2 & 1.38 & 0.462 & $\S 4.4$ \\
11 & 7 & 4 & 2 & 1.45 & 0.45 & $\S 4.4$ \\
9 & 7 & 3 & 2 & 1.56 & 0.44 & $\S 4.4$ \\
7 & 7 & 2 & 2 & 1.71 & 0.43 & $\S 4.4$ \\
14 & 12 & 5 & 3 & 1.64 & 0.43 & $\S 4.5$ \\
12 & 12 & 4 & 3 & 1.75 & 0.42 & $\S 4.5$ \\
5 & 7 & 1 & 3 & 1.80 & 0.40 & $\S 4.4$ \\
8 & 12 & 2 & 3 & 2.13 & 0.38 & $\S 4.5$ \\
11 & 16 & 3 & 1 & 2.36 & 0.36 & $\S 4.1$ \\
6 & 12 & 1 & 3 & 2.50 & 0.33 & $\S 4.5$ \\
7 & 16 & 1 & 1 & 3.14 & 0.29 & $\S 4.1$ \\
8 & 32 & 1 & 1 & 4.88 & 0.25 & $\S 4.2$ \\
9 & 62 & 1 & 1 & 7.78 & 0.22 & $\S 4.2$ \\
\hline
\end{tabular}

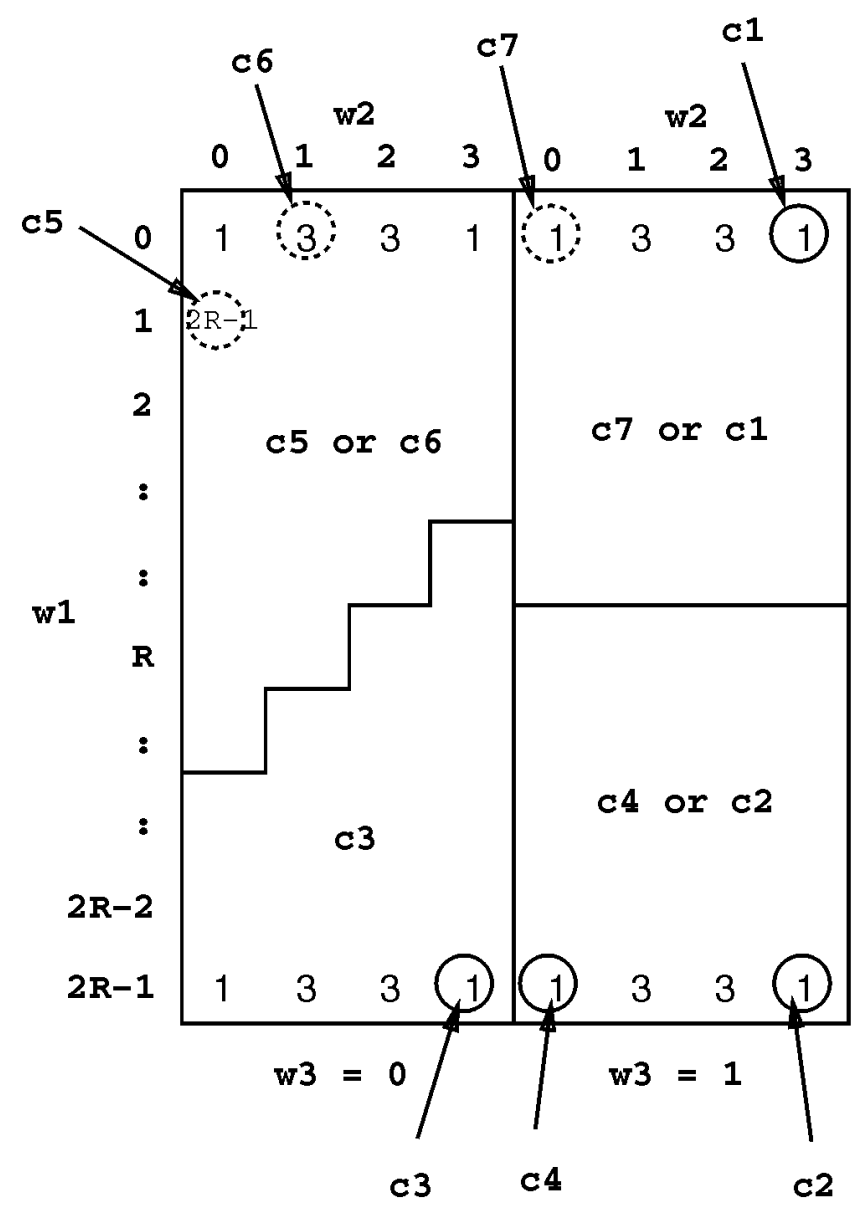

Fig. 6. Three-dimensional array showing a family of piecewise constant codes as the $(2 R+3,4, R)^{+}$-covering codes. 
TABLE 7

BEST CHOICES OF SAND $T$ BASED ON EXISTING AND SINGLE-WEIGHT-EXTENDED COVERING CODES

\begin{tabular}{|c||c|c|c|c|c|c|c|}
\hline$n$ & $K$ & $R$ & $c$ & $K^{\prime}$ & $s$ & $t$ & ref. \\
\hline \hline$m$ & 2 & $m / 2$ & 1 & - & $1+1 / m$ & 0.50 & \\
odd $n$ & - & $(n-3) / 2$ & - & 4 & $1+4 / n$ & $0.5-\frac{1}{2 n}$ & $\S 5.2$ \\
19 & - & 8 & - & 4 & 1.21 & 0.474 & $\S 5.2$ \\
17 & - & 7 & - & 4 & 1.24 & 0.471 & $\S 5.2$ \\
15 & - & 6 & - & 4 & 1.27 & 0.467 & $\S 5.2$ \\
13 & - & 5 & - & 4 & 1.31 & 0.462 & $\S 5.2$ \\
11 & - & 4 & - & 4 & 1.36 & 0.45 & $\S 5.2$ \\
9 & - & 3 & - & 4 & 1.44 & 0.44 & $\S 5.2$ \\
7 & - & 2 & - & 4 & 1.57 & 0.43 & $\S 5.2$ \\
12 & 12 & 4 & 3 & - & 1.75 & 0.42 & $\S 4.5$ \\
5 & 7 & 1 & 3 & - & 1.80 & 0.40 & $\S 4.4$ \\
8 & 12 & 2 & 3 & - & 2.13 & 0.38 & $\S 4.5$ \\
11 & 16 & 3 & 1 & - & 2.36 & 0.36 & $\S 4.1$ \\
6 & 12 & 1 & 3 & - & 2.50 & 0.33 & $\S 4.5$ \\
7 & 16 & 1 & 1 & - & 3.14 & 0.29 & $\S 4.1$ \\
8 & 32 & 1 & 1 & - & 4.88 & 0.25 & $\S 4.2$ \\
9 & 62 & 1 & 1 & - & 7.78 & 0.22 & $\S 4.2$ \\
\hline
\end{tabular}

for $1 \leq i \leq R, C_{i} \in C$.

For example, consider a code $C=\left\{C_{1}=(1111), C_{2}=(0111), C_{3}\right.$ $\left.=(0110), C_{4}=(0101), C_{5}=(0011), C_{6}=(1000)\right\}$. It can be verified from Table 8 that this code is a $(4,6,1)^{*}$-covering code.

Clearly, an $\left(n, K^{\prime}, R\right)^{+}$-covering code is also an $\left(n, K^{\prime}+n, R\right)^{*}$ covering code, but not vice versa. We will use $K^{\prime \prime}$ throughout this section to denote the total number of codewords. Note that the code may not contain all weight- 1 vectors as codewords. However, in our computer search, we minimize $K^{\prime \prime}$ first, given $n$ and $R$, then maximize the total number of weight-1 vectors among all minimum- $K^{\prime \prime}$ solutions. We were able to find a minimum- $K^{\prime \prime}$ solution with all $n$ weight- 1 vectors included as codewords for all cases listed below.

Given an $\left(n, K^{\prime \prime}, R\right)^{*}$-composition-extended covering code $C$ and any $n$-bit vector $V$, we will redefine $f_{t}(n)$ and $f_{s}(n)$ as the time and associated space overheads, respectively, to find the set of codewords $C_{1}, \cdots, C_{i+1}$ and its precomputed corresponding partial sums such that $V=C_{1} \odot$ $C_{2} \odot \cdots \odot C_{i+1}$ where $0 \leq i \leq R$.

THEOREM 6. Given an $\left(n, K^{\prime \prime}, R\right)^{*}$-covering code, we can construct an algorithm to derive the partial sum $\operatorname{Psum}(A, I)$ in time $T \approx\left(R+f_{t}(n)+1\right) \frac{m}{n}$ and in space $S \approx K^{\prime \prime} \frac{m}{n}+f_{s}(n)$.

PROOF. We first show that given an $\left(m, K^{\prime \prime}, R\right)^{*}$-covering code $C$, we can construct an algorithm to derive the partial sum $\operatorname{Psum}(A, I)$ in time $T=R+f_{t}(m)+1$ and in space $S=K^{\prime \prime}+f_{s}(m)$. We will precompute and store the $K^{\prime \prime}$ partial sums of $A$ that correspond to the $K^{\prime \prime}$ codewords. Given an index subset $I$ at run time, let $V=\operatorname{mask}(I)$. By Definition 3, we can assume $V=C_{1} \odot$ $C_{2} \odot \cdots \odot C_{x+1}$ where $0 \leq x \leq R$ and $C_{x} \in C$. Let $I_{i}=$ $\operatorname{support}\left(C_{i}\right)$ for all $1 \leq i \leq x+1$. By Lemma 5 , we can derive $\operatorname{Psum}(A, I)$ by combining $\operatorname{Psum}\left(A, I_{i}\right)$ s through addition or subtraction for all $1 \leq i \leq x+1$. This requires an overhead of $f_{t}(m)+R+1$ in time and $f_{s}(m)+K^{\prime \prime}$ in space. The rest of the proof is similar to that of Theorem 2 by applying the time and space overhead to each block of $A$ of size $n$.
TABLE 8

THE $(4,6,1)^{*}$-COVERING CODE

\begin{tabular}{|c|c|c|c|}
\hline weight & Vector & the composition & min distance \\
\hline \hline \multirow{3}{*}{1} & $(0001)$ & $(0111) \odot(0110)$ & 1 \\
& $(0010)$ & $(0111) \odot(0101)$ & 1 \\
& $(0100)$ & $(0111) \odot(0011)$ & 1 \\
& $(1000)$ & itself & 0 \\
\hline \multirow{5}{*}{2} & $(0011)$ & itself & 0 \\
& $(0110)$ & itself & 0 \\
& $(1100)$ & $(1111) \odot(0011)$ & 1 \\
& $(1001)$ & $(1111) \odot(0110)$ & 1 \\
& $(0101)$ & itself & 0 \\
& $(1010)$ & $(1111) \odot(0101)$ & 1 \\
\hline \multirow{3}{*}{3} & $(0111)$ & itself & 0 \\
& $(1110)$ & $(1000) \odot(0110)$ & 1 \\
& $(1101)$ & $(1000) \odot(0101)$ & 1 \\
& $(1011)$ & $(1000) \odot(0011)$ & 1 \\
\hline 4 & $(1111)$ & itself & 0 \\
\hline
\end{tabular}

\subsection{Lower Bounds on $K^{\prime \prime}$}

LEMMA 7. Let $S_{i} \in\{+1,-1\}, 1 \leq i \leq x$. If $C_{1} \odot C_{2} \odot \ldots \odot C_{x}=V$ $\neq \perp$, then there exists a set of $S_{i}$ such that $S_{1} C_{1}+S_{2} C_{2}+$ $\cdots+S_{x} C_{x}=V$, where the addition is bit-wise.

ProOF. By Definition 3 and the fact that $V \neq \perp$, we have $C_{1} \odot C_{2} \in\left\{C_{1}+C_{2},-C_{1}+C_{2}, C_{1}-C_{2}\right\}$. By applying the same argument to the sequence $C_{1} \odot C_{2} \odot \cdots \odot C_{x}$, the proof follows.

LEMMA 8. Let $\pi$ be a permutation function of $\{1,2, \cdots, x\}$. If $C_{1}$ $\odot C_{2} \odot \ldots \odot C_{x}=V \neq \perp$ and $C_{\pi_{(1)}} \odot C_{\pi_{(2)}} \odot \ldots \odot C_{\pi_{(x)}}$ $=V^{\prime} \neq \perp$, then $V=V^{\prime}$.

ProOF. Let $S_{i} \in\{+1,-1\}$ be the sign associated with $C_{i}$ in order to derive $V$, Lemma 7. That is, $\sum_{i=1}^{x} S_{i} C_{i}=V$. Let $S$ be the ordered set $\left\{S_{1}, S_{2}, \cdots, S_{x}\right\}$. Assume that $V \neq V^{\prime}$. Then, there exists a new ordered set $S^{\prime}=\left\{S_{1}^{\prime}, S_{2}^{\prime}, \ldots, S_{x}^{\prime}\right\}$ such that $\sum_{i=1}^{x} S_{i}^{\prime} C_{i}=V^{\prime}$ and $S^{\prime} \neq S$ (i.e., $S_{i}^{\prime} \neq S_{i}$ for some $i \in\{1,2, \cdots, x\})$. The set $S^{\prime}$ can be derived from the set of $S$ by changing all different $\left(S_{i}, S_{i}^{\prime}\right)$-pairs.

Note, however, that every change of sign from $S_{i}$ to $S_{i}^{\prime}$ will result in a "distance-2" or "distance-0" move of all digits in $V$. More specifically, the $j$ th digit with value $v$ will be changed to one of $\{v+2, v, v-2\}$, depending on the $j$ th bit of $C_{i}$. Thus, a digit which is even (positive, zero, or negative) remains even due to the changes of signs. Similarly, a digit which is odd (positive or negative) remains odd. For instance, a 0digit in $V$ will be changed to one in $\{-2,0,2\}$ due to one sign change, while a 1-digit will be changed to one in $\{-1,1,3\}$. Since 0 is the only valid even digit of any defined vector and 1 is the only valid odd digit of any defined vector, $V=V^{\prime}$.

In the above proof, it is possible that $V=V^{\prime}$ while $S \neq S^{\prime}$. In this case, there must be some number of codewords which compose to an all-0 vector. 
TABLE 9

Best Obtained Choices of S And $T$ Based on All Techniques

\begin{tabular}{|c||c|c|c|c|c|c|c|c|}
\hline$n$ & $K$ & $R$ & $c$ & $K^{\prime}$ & $K^{\prime \prime}$ & $s$ & $t$ & reference \\
\hline \hline$m$ & 2 & $m / 2$ & 1 & - & - & $1+1 / m$ & 0.50 & \\
odd $n$ & - & $(n-3) / 2$ & - & 4 & - & $1+4 / n$ & $0.5-\frac{1}{2 n}$ & $\S 5.2$ \\
19 & - & 8 & - & 4 & - & 1.21 & 0.474 & $\S 5.2$ \\
17 & - & 7 & - & 4 & - & 1.24 & 0.471 & $\S 5.2$ \\
15 & - & 6 & - & 4 & - & 1.27 & 0.467 & $\S 5.2$ \\
13 & - & 5 & - & 4 & - & 1.31 & 0.462 & $\S 5.2$ \\
11 & - & 4 & - & 4 & - & 1.36 & 0.45 & $\S 5.2$ \\
9 & - & 3 & - & 4 & - & 1.44 & 0.44 & $\S 5.2$ \\
7 & - & 2 & - & 4 & - & 1.57 & 0.43 & $\S 5.2$ \\
12 & 12 & 4 & 3 & - & - & 1.75 & 0.42 & $\S 4.5$ \\
5 & 7 & 1 & 3 & - & - & 1.80 & 0.40 & $\S 4.4$ \\
8 & - & 2 & - & - & 15 & 1.88 & 0.38 & $\S 6$ \\
6 & - & 1 & - & - & 13 & 2.17 & 0.33 & $\S 6$ \\
7 & - & 1 & - & - & 21 & 3.00 & 0.29 & $\S 6$ \\
8 & - & 1 & - & - & 29 & 3.63 & 0.25 & $\S 6$ \\
9 & - & 1 & - & - & 45 & 5.00 & 0.22 & $\S 6$ \\
\hline
\end{tabular}

THEOREM 9. Any $\left(n, K^{\prime \prime}, R\right)^{*}$-covering code must have

$$
\sum_{i=1}^{R+1}\left(\begin{array}{c}
K^{\prime \prime} \\
i
\end{array}\right) \geq 2^{n}-1
$$

PROOF. Follows from Lemma 8.

COROLlary 10. Any $\left(n, K^{\prime \prime}, 1\right)^{*}$-covering code must have

$$
\frac{K^{\prime \prime}\left(K^{\prime \prime}+1\right)}{2} \geq 2^{n}-1 \text {. }
$$

COROLlary 11. Any $\left(n, K^{\prime \prime}, 2\right)^{*}$-covering code must have

$$
\frac{K^{\prime \prime}\left(K^{\prime \prime 2}+5\right)}{6} \geq 2^{n}-1 \text {. }
$$

\subsection{Some Useful Composition-Extended Covering Codes}

To find "good" composition-extended covering codes, we implemented a computer search program based on various heuristics to search in selected subspace than an exhaustive one. In the following, we list the best composition-extended covering codes that we found so far; each is a result of a run of at least one day on a typical workstation. It may be possible to improve these codes by having longer runs.

\subsubsection{The $(6,13,1)^{*}$-Covering Code}

$$
C=\{1,2,4,6,8,16,25,32,34,36,47,55,62\} .
$$

This code improves from previous $K^{\prime \prime}=K-c+n=15$ (due to $(6,12,1)$-covering code in Section 4.5$)$ to 13 . The number of weight- 1 codewords is 6 . The lower bound on $K^{\prime \prime}$ is 11 , by Corollary 10.

\subsubsection{The $(7,21,1)^{*}$-Covering Code}

$$
\begin{gathered}
C=\{1,2,4,8,16,24,32,33,38,39,64, \\
72,80,91,93,94,95,122,123,124,125\} .
\end{gathered}
$$

This code improves from previous $K^{\prime \prime}=22$ (due to $(7,16,1)$ Hamming code in Section 4.1) to 21. The number of weight-1 codewords is 7. The lower bound on $K^{\prime \prime}$ is 16 , by Corollary 10 .

\subsubsection{The $(8,29,1)^{*}$-Covering Code}

$C=\{1,2,3,4,8,16,17,18,19,32,64,76,100,108,128,129$, $130,131,144,145,146,159,183,187,191,215,219,243,251\}$.

This code improves from previous $K^{\prime \prime}=39$ (due to $(8,32$, 1 )-covering code in Section 4.2) to 29. The number of weight- 1 codewords is eight. The lower bound on $K^{\prime \prime}$ is 23 , by Corollary 10.

\subsubsection{The $(9,45,1)^{*}$-Covering Code \\ $C=\{1,2,3,4,8,16,17,18,19,32,36,40,44,64$, $68,96,100,104,128,132,136,140,160,232,236$, $256,257,258,259,272,273,274,287,347,351$, $383,439,443,447,467,471,475,479,499,503\}$.}

This code improves from previous $K^{\prime \prime}=70$ (due to $(9,62$, 1 )-covering code in Section 4.2) to 45 . The number of weight- 1 codewords is nine. The lower bound on $K^{\prime \prime}$ is 32 , by Corollary 10.

\subsubsection{The $(8,15,2)^{*}$-Covering Code$$
C=\{1,2,3,4,8,16,32,33,34,64,115,128,191,204,255\} .
$$

This code improves from previous $K^{\prime \prime}=17$ (due to $(8,12,2$ )covering code in Section 4.5) to 15. The number of weight-1 vectors is eight. The lower bound on $K^{\prime \prime}$ is 12 , by Corollary 11.

\subsection{Results}

Table 9 summarizes the best $(s, t)$-pairs obtained based on the previous Table 7 and the new codes given in this section. Fig. 7 shows three sets of data points corresponding to the $(s, t)$-pairs derived from the existing covering codes, new single-weight-extended covering codes, and new composition-extended covering codes. Fig. 1 shows the best $(s, t)$-pairs combining results from all three types of covering codes, i.e., corresponding to Table 9. Note that in Fig. 7, the data points for covering codes and those for single-weight-extended covering codes do not overlap. For the composition-extended covering codes, the curve stops at $s=5$ because the next $(s, t)$ point requires searching a good 


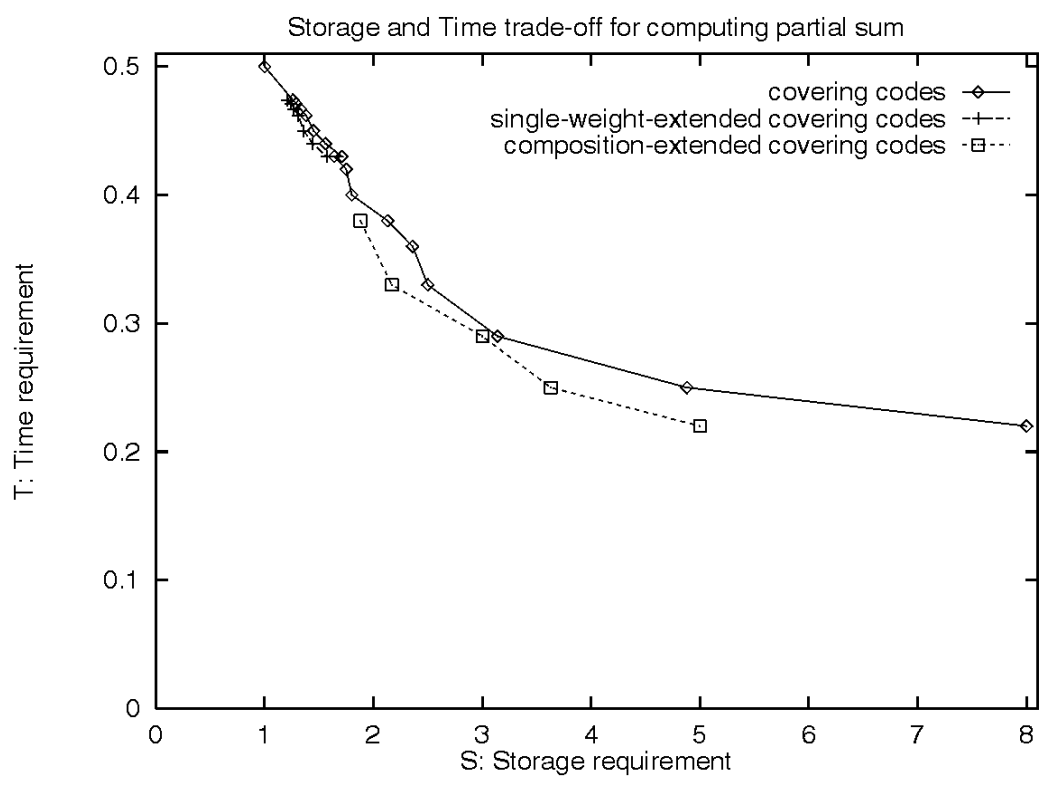

Fig. 7. Three types of $(s, t)$ data points for computing partial sum.

$\left(10, K^{\prime \prime}, 1\right)^{*}$-covering code, a complicated search for little gain in time, from 0.22 for $n=9$ to 0.2 for $n=10$.

\section{Partial Sums for Multidimensional Arrays}

In this section, we will generalize the one-dimensional partialsum algorithm to the $d$-dimensional case. Assume $A$ is a $d$ dimensional array of form $m_{1} \times \cdots \times m_{d}$ and let $m=\prod_{i=1}^{d} m_{i}$ be the total size of $A$. Let $M$ be the index domain of $A$. Let $D=$ $\{1, \ldots, d\}$ be the set of dimensions. For each $i \in D$, let $I_{i}$ be an arbitrary subset of $\left\{0, \ldots, m_{i}-1\right\}$ specified by the user at query time. Also let $I=\left\{\left(x_{1}, \ldots, x_{d}\right) \mid(\forall i \in D)\left(x_{i} \in I_{i}\right)\right\}$. That is, $I=I_{1} \times \cdots \times I_{d}$ and $I \subset M$.

Given $A$ in advance and $I$ during the query time, we are interested in getting partial sum of $A$, specified by $I$ as:

$$
\operatorname{Psum}(A, I)=\sum_{\forall\left(x_{1}, \ldots x_{d}\right) \in I} A\left[x_{1}, \ldots, x_{d}\right] .
$$

\subsection{A Motivating Example}

Before giving the general $d$-dimensional algorithm and theorem, we first give a motivating two-dimensional example. Assume $A$ is a two-dimensional array of form $5 \times 5$. Also assume that we are applying the $(5,7,1)$-covering code, which is also a $(5,9,1)^{+}$-single-weight-extended covering code, to each dimension. Denote the nine codewords by $C_{0}$ through $C_{8}$, consistent with the order in Table 2. The index look-up table, denoted by $X$, is still the same as that for the one-dimensional case, Table 3 . On the other hand, the partial-sum look-up table will be extended from Table 2 (which has nine entries) to a two-dimensional table, denoted by $P$, of $9 \times 9$ entries. Then, we will let $P[i, j]$ contain the precomputed partial sum $\operatorname{Psum}\left(A, \operatorname{support}\left(C_{i}\right) \times \operatorname{support}\left(C_{j}\right)\right)$.

For convenience, we will view each entry of $X$ as a set of (sign, index) pairs. Assume given $I_{1}=\{3,4\}$ and $I_{2}=\{1,3,4\}$ at query time. We use mask $\left(I_{1}\right)$, which is $(00011)=3$, as an index to the index look-up table $X$ and obtain $X\left[\operatorname{mask}\left(I_{1}\right)\right]=$ $\{(+1,3),(+1,4)\}$. Also, we use mask $\left(I_{2}\right)$, which is $(01011)=11$, as an index to the same index look-up table $X$ and obtain $X\left[\operatorname{mask}\left(I_{2}\right)\right]=\{(+1,6),(-1,0)\}$. We will show later that $\operatorname{Psum}(A, I)$ can be computed as follows.

$$
\operatorname{Psum}(A, I)=\sum_{\forall\left(s_{i}, x_{i}\right) \in X\left[\operatorname{mack}\left(I_{i}\right)\right]}\left\{\left(\prod_{i \in D} s_{i}\right) P\left[x_{1}, \ldots, x_{d}\right]\right\} .
$$

Following this, we have $\operatorname{Psum}(A, I)=P[3,6]+P[4,6]-P[3$, $0]-P[4,0]$ for our example. Intuitively, the final partial sum $\operatorname{Psum}(A, I)$ is derived from combination of additions and subtractions of all "relevant entries" in $P$, where the "relevant entries" are Cartesian products of different entries indexed by $X\left[\operatorname{mask}\left(I_{i}\right)\right]$. Table 10 shows the precomputed partial sums corresponding to the four terms on the right hand side of the formula. Fig. 8 gives a pictorial view corresponding to the formula. In the figure, 1 means a selected value.

\subsection{The Main Theorem}

We are now ready to prove a lemma for the general case of the above example.

LEMMA 12. Let $B$ be a d-dimensional array of form $n \times \cdots \times$ $n$, and let Psum $(B, I)$ be the partial-sum query. Then, given an $\left(n, K^{\prime \prime}, R\right)^{*}$-covering code, we can construct an algorithm to derive Psum $(B, I)$ for any $I$ in time $T=$ $(R+1)^{d}+f_{t}(n) d$ and in space $S=K^{\prime \prime d}+f_{s}(n)$.

TABLE 10

EXAMPLES OF INDEXED PARTIAL SUMS IN THE PARTIAL-SUM LOOK-UP TABLE

\begin{tabular}{|c|c|}
\hline index & partial sum \\
\hline \hline$(3,6)$ & $A[3,0]+A[3,1]+A[3,3]+A[3,4]$ \\
\hline$(4,6)$ & $A[4,0]+A[4,1]+A[4,3]+A[4,4]$ \\
\hline$(3,0)$ & $A[3,0]$ \\
\hline$(4,0)$ & $A[4,0]$ \\
\hline
\end{tabular}




$\left.\left.\begin{array}{lllll}0 & 0 & 0 & 0 & 0 \\
0 & 0 & 0 & 1 & 1 \\
0 & 0 & 0 & 0 & 0 \\
0 & 0 & 0 & 1 & 1 \\
0 & 0 & 0 & 1 & 1\end{array}\right]=\begin{array}{lllll}0 & 0 & 0 & 1 & 0 \\
0 & 0 & 0 & 1 & 0 \\
0 & 0 & 0 & 0 & 0 \\
0 & 0 & 0 & 1 & 0 \\
0 & 0 & 0 & 1 & 0\end{array}\right]+$\begin{tabular}{lllll|}
0 & 0 & 0 & 0 & 1 \\
0 & 0 & 0 & 0 & 1 \\
0 & 0 & 0 & 0 & 0 \\
0 & 0 & 0 & 0 & 1 \\
0 & 0 & 0 & 0 & 1
\end{tabular}

$\left.-\begin{array}{lllll}0 & 0 & 0 & 1 & 0 \\ 0 & 0 & 0 & 0 & 0 \\ 0 & 0 & 0 & 0 & 0 \\ 0 & 0 & 0 & 0 & 0 \\ 0 & 0 & 0 & 0 & 0\end{array}\right]-\left[\begin{array}{lllll|}0 & 0 & 0 & 0 & 1 \\ 0 & 0 & 0 & 0 & 0 \\ 0 & 0 & 0 & 0 & 0 \\ 0 & 0 & 0 & 0 & 0 \\ 0 & 0 & 0 & 0 & 0 \\ \hline\end{array}\right.$

Fig. 8. A pictorial view of $P \operatorname{sum}(A, I)=P[3,6]+P[4,6]-P[3,0]-P[4,0]$.

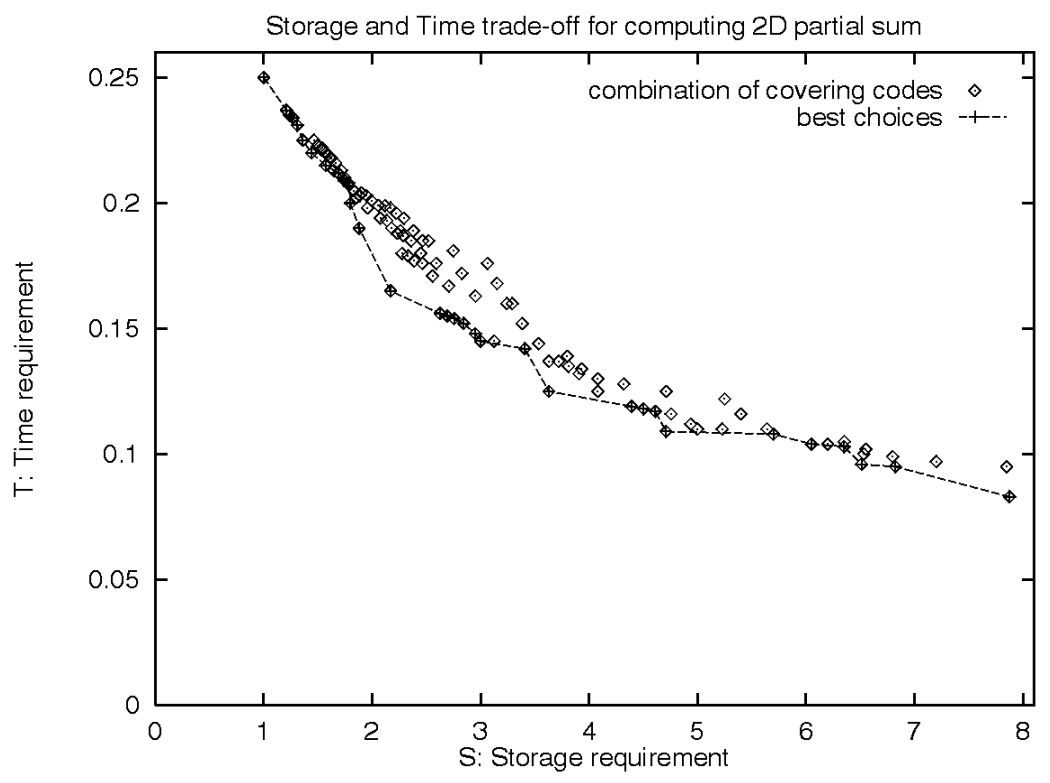

Fig. 9. The best $(s, t)$ data points for computing two-dimensional partial sum.

ProOF. Denote the set of $K^{\prime \prime}$ codewords by $C=\left\{C_{0}, C_{1}, \ldots\right.$, $\left.C_{K^{\prime \prime}-1}\right\}$. Let $J_{i}=\operatorname{support}\left(C_{i}\right)$. We first construct a $d$ dimensional partial-sum look-up table, of form $K^{\prime \prime} \times$ $\cdots \times K^{\prime \prime}$. An entry indexed by $\left(x_{1}, \cdots, x_{d}\right)$ in the table will contain precomputed result for $\operatorname{Psum}(B, J)$, where $J=J_{x_{1}} \times \cdots \times J_{x_{d}}$. Given $I$ at query time, let $I=I_{1} \times \cdots$ $\times I_{d}$. Note that, in the one-dimensional domain, each $I_{i}$ can be derived by combining up to $R+1$ existing partial sums. Through an inductive proof, one can show that $I$ can be derived by combining up to $(R+1)^{d}$ existing partial sums from the partial-sum look-up table. For each dimension, a time overhead of $f_{t}(n)$ is needed to derive the index of that dimension to the partial-sum look-up table. Thus, the overall time is $T=(R+1)^{d}+f_{t}(n) d$. For the space overhead, the partialsum look-up table is of size $K^{\prime \prime d}$ and the index look-up table is of size $f_{s}(n)$. Since we apply the same covering code to all $d$ dimensions, there is only one index lookup table needed. Thus, the overall space overhead is $S=K^{\prime \prime d}+f_{s}(n)$.
As in the one-dimensional case, we will now partition array $A$ into blocks of form $n \times \cdots \times n$ and apply covering codes to each block (using the above lemma) in order to derive better space overheads. The proof of the following theorem is straightforward:

THEOREM 13. Given an $\left(n, K^{\prime \prime}, R\right)^{*}$-covering code, we can construct an algorithm to derive the d-dimensional partial sum $\operatorname{Psum}(A, I)$ in time $T \approx\left(\frac{R+1}{n}\right)^{d} m+d f_{t}(n) \frac{m}{n^{d}}$ and in space $S \approx\left(\frac{K^{\prime \prime}}{n}\right)^{d} m+f_{s}(n)$.

The above theorem assumes that the same covering code is applied to all dimensions of each block and, thus, each block is of form $n \times \cdots \times n$. In general, one can apply different covering codes to different dimensions and obtain a wider range of space-time trade-offs. In this case, the length of each side of the block will be tailored to the length of each covering code applied.

COROLLARY 14 . Given an $\left(n, K^{\prime \prime}, R\right)^{*}$-covering code, we can construct an algorithm to derive the d-dimensional partial sum $\operatorname{Psum}(A, I)$ in time $T \approx\left(\frac{R+1}{n}\right)^{\alpha}\left(\frac{1}{2}\right)^{d-\alpha} m+d f_{t}(n) \frac{m}{n^{\alpha}}$ and in space $S \approx\left(\frac{K^{\prime \prime}}{n}\right)^{\alpha}\left(1+\frac{1}{m}\right)^{d-\alpha} m+f_{s}(n)$. 
ProOF. Apply an $\left(n, K^{\prime \prime}, R\right)^{*}$-composition-extended covering code to $\alpha$ dimensions and the $\left(m_{i}, m_{i}+1,\left\lceil m_{i} / 2\right\rceil\right)^{+}-$ single-weight-extended covering code to the remaining $d-\alpha$ dimensions. The proof completes by noticing that the latter code has $(s, t) \approx(1,0.5)$.

\subsection{Results}

Fig. 9 shows various $(s, t)$ data points for computing twodimensional partial sum based on combination of onedimensional $(s, t)$ data points from Table 9 . The best $(s, t)$ data points are joined together by a curve. Note the leftmost $(s, t)$ data point has been changed from $(1,0.5)$ in Fig. 1 to $(1,0.25)$ in this figure.

\section{SUMmARY}

Partial-sum queries obtain the summation over specified cells of a data cube. In this paper, we established the connection between the covering problem [12] in the theory of error-correcting codes and the partial-sum problem. We use this connection to apply four known covering codes from [12], [5], and [4] to the partial-sum problem to obtain algorithms with various space-time trade-offs. We then modified the requirements on covering codes to better reflect the partial-sum problem and devise new covering codes with respect to the new requirements. As a result, we develop new algorithms with better space-time trade-offs. For example, using these algorithms, with 44 percent additional storage, the query response time can be improved by about 12 percent; by roughly doubling the storage requirement, the query response time can be improved by about 34 percent.

\section{ACKNOWLEDGMENTS}

This research was supported in part by U.S. National Science Foundation Young Investigator Award CCR-9457811 and by the Sloan Research Fellowship.

\section{REFERENCES}

[1] S. Agrawal, R. Agrawal, P.M. Deshpande, A. Gupta, J.F. Naughton, R. Ramakrishnan, and S. Sarawagi, "On the Computation of Multidimensional Aggregates," Proc. 22nd Int'l Conf. Very Large Databases, pp. 506-521, Mumbai (Bombay), India, Sept. 1996.

[2] L.S. Colby, R.L. Cole, E. Haslam, N. Jazayeri, G. Johnson, W.J. McKenna, L. Schumacher, and D. Wilhite, "Red Brick Vista: Aggregate Computation and Management," Proc. 14th Int'l Conf. Data Eng., pp. 174-177, 1998.

[3] G.D. Cohen, I. Honkala, S. Litsyn, and A.C. Lobstein, Covering Codes. Elsevier, 1977.

[4] G.D. Cohen, S. Litsyn, A.C. Lobstein, and H.F. Mattson Jr., "Covering Radius 1985-1994," J. Applicable Algebra in Eng., Comm., and Computing, special issue, vol. 8, no. 3, 1997.

[5] G.D. Cohen, A.C. Lobstein, and N.J.A. Sloane, "Further Results on the Covering Radius of Codes," IEEE Trans. Information Theory, vol. 32, no. 5, pp. 680-694, Sept. 1986.

[6] M.C. Chen and L.P. McNamee, "The Data Model and Access Method of Summary Data Management," IEEE Trans. Knowledge and Data Eng., vol. 1, no. 4, pp. 519-529, 1989.

[7] E.F. Codd, "Providing OLAP (On-Line Analytical Processing) to User-Analysts: An IT Mandate," technical report, E.F. Codd and Assoc., 1993.

[8] S. Chaudhuri and K. Shim, "Including Group-By in Query Optimization," Proc. 20th Int'l Conf. Very Large Databases, pp. 354-366, Santiago, Chile, Sept. 1994.
[9] J. Gray, A. Bosworth, A. Layman, and H. Pirahesh, "Data Cube: A Relational Aggregation Operator Generalizing Group-By, CrossTabs, and Sub-Totals," Proc. 12th Int'l Conf. Data Eng., pp. 152-159, 1996.

[10] A. Gupta, V. Harinarayan, and D. Quass, "Aggregate-Query Processing in Data Warehousing Environments," Proc. Eighth Int'l Conf. Very Large Databases (VLDB), pp. 358-369, Zurich, Switzerland, Sept. 1995.

[11] H. Gupta, V. Harinarayan, A. Rajaraman, and J.D. Ullman, "Index Selection for OLAP," Proc. 13th Int'l Conf. Data Eng., Birmingham, U.K., Apr. 1997.

[12] R.L. Graham and N.J.A. Sloane, "On the Covering Radius of Codes," IEEE Trans. Information Theory, vol. 31, no. 3, pp. 385-401, May 1985.

[13] C.-T. Ho, R. Agrawal, N. Megiddo, and R. Srikant, "Range Queries in OLAP Data Cubes," Proc. ACM SIGMOD Conf. Management of Data, Tucson, Ariz., May 1997.

[14] V. Harinarayan, A. Rajaraman, and J.D. Ullman, "Implementing Data Cubes Efficiently," Proc. ACM SIGMOD Conf. Management of Data, June 1996.

[15] T. Johnson and D. Shasha, "Hierarchically Split Cube Forests for Decision Support: Description and Tuned Design," working paper, 1996.

[16] Special issue on materialized views and data warehousing, D. Lomet, ed., IEEE Data Eng. Bulletin, vol. 18, no. 2, June 1995.

[17] Z. Michalewicz, Statistical and Scientific Databases. Ellis Horwood, 1992.

[18] The OLAP Council, MD-API the OLAP Application Program Interface Version 0.5 Specification, Sept. 1996.

[19] A. Shukla, P.M. Deshpande, J.F. Naughton, and K. Ramasamy, "Storage Estimation for Multidimensional Aggregates in the Presence of Hierarchies," Proc. 22nd Int'l Conf. Very Large Databases, pp. 522-531, Mumbai (Bombay), India, Sept. 1996.

[20] B. Salzberg and A. Reuter, "Indexing for Aggregation," working paper, 1996.

[21] J. Srivastava, J.S.E. Tan, and V.Y. Lum, "TBSAM: An Access Method for Efficient Processing of Statistical Queries," IEEE Trans. Knowledge and Date Eng., vol. 1, no. 4, 1989.

[22] W.P. Yan and P. Larson, "Eager Aggregation and Lazy Aggregation," Proc. Eighth Int'l Conf. Very Large Databases (VLDB), pp. 345357, Zurich, Switzerland, Sept. 1995.

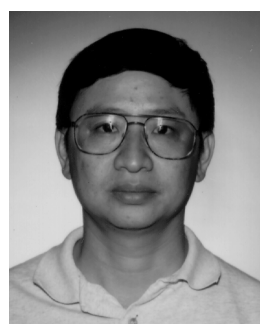

Ching-Tien Ho received a BS degree in electrical engineering from the National Taiwan University in 1979 and MS, MPhil, and PhD degrees in computer science from Yale University in 1985, 1986, and 1990, respectively.

He joined the IBM Almaden Research Center as a research staff member in 1989. He was manager of the Foundations of Massively Parallel Computing Group from 1994 to 1996, where he led the development of collective communication, as part of IBM MPL and MPI, for IBM SP-1 and SP-2 parallel systems. His primary research interests include communication issues for interconnection networks, algorithms for collective communications, graph embeddings, fault tolerance, and parallel algorithms and architectures. His current interests are data mining and on-line analytical processing. He has published more than 80 journal and conference papers in these areas.

Dr. Ho is a co-recipient of the 1986 "Outstanding Paper Award" of the International Conference on Parallel Processing. He has received an IBM Outstanding Innovation Award, two IBM Outstanding Technical Achievement Awards, and four IBM Plateau Invention Achievement Awards. He has 11 patents granted or pending. He is on the Editorial Board of the IEEE Transactions on Parallel and Distributed Systems. $\mathrm{He}$ is one of program vice-chairs for the 1998 International Conference on Parallel Processing. He has worked on program committees of many parallel processing conferences and workshops. He is a member of the ACM, the IEEE, and the IEEE Computer Society. 


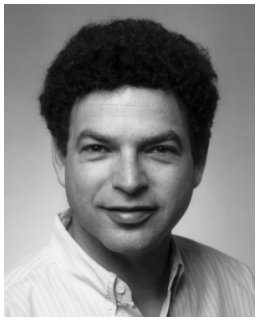

Jehoshua Bruck received the BSc and MSc degrees in electrical engineering from the Technion, Israel Institute of Technology, in 1982 and 1985 , respectively, and the $\mathrm{PhD}$ degree in electrical engineering from Stanford University in 1989.

$\mathrm{He}$ is a professor of computation and neural systems and electrical engineering at the California Institute of Technology. His research interests include parallel and distributed computing, fault-tolerant computing, error-correcting codes, computation theory, and biological systems. Dr. Bruck has extensive industrial experience, including serving as manager of the Foundations of Massively Parallel Computing Group at the IBM Almaden Research Center from 1990 to 1994, a research staff member at the IBM Almaden Research Center from 1989 to 1990, and a researcher at the IBM Haifa Science Center from 1982 to 1985.

Dr. Bruck is the recipient of a 1997 IBM Partnership Award, a 1995 Sloan Research Fellowship, a 1994 U.S. National Science Foundation Young Investigator Award, six IBM Plateau Invention Achievement Awards, 11992 IBM Outstanding Innovation Award for his work on "harmonic analysis of neural networks," and a 1994 IBM Outstanding Technical Achievement Award for his contributions to the design and implementation of the SP-1, the first IBM scalable parallel computer $\mathrm{He}$ has published more than 130 journal and conference papers in his areas of interests and he holds 21 patents. Dr. Bruck is a senior member of the IEEE and a member of the Editorial Board of the IEEE Transactions on Parallel and Distributed Systems.

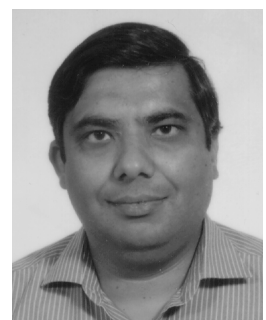

Rakesh Agrawal received the MS and $\mathrm{PhD}$ degrees in computer science from the University of Wisconsin, Madison in 1983. He also has a $\mathrm{BE}$ degree in electronics and communication engineering from the University of Roorkee, Roorkee, India, and a two-year postgraduate diploma in industrial engineering from NITIE, Bombay, India. He is the project leader and manager of the Quest project on Data Mining and Decision Support Technologies at the IBM Almaden Research Center, San Jose, California. From 1983 to 1989, he was with AT\&T Bell Laboratories, Murray Hill, New Jersey, where he was a member of the technical staff in the Computing Systems Research Laboratory. He has been with the IBM Almaden Research Center since January 1990.

Dr. Agrawal is currently a program chair for the Fourth International Conference on Knowledge Discovery and Data Mining (KDD-98) and a trustee of the VLDB Endowment. He is also an editor of the ACM Transactions on Database Systems, VLDB Journal, and the Data Mining and Knowledge Discovery Journal. He has been the chair of the IEEE Technical Committee on Data Engineering, a program chair for the 19th International Conference on Very Large Databases (VLDB93), a program chair for the Second International Symposium on Databases in Parallel and Distributed Systems (DPDS-90), an editor of the IEEE Transactions on Knowledge and Data Engineering, and editor of the IEEE Transactions on Parallel and Distributed Systems, and an associate editor of the IEEE Data Engineering Bulletin. He has published extensively in technical journals and conferences. He has also authored a book on Programming in ANSI C.

Dr. Agrawal is a member of the IBM Academy of Technology and a Research Division Master Inventor. He has received IBM's Outstanding Innovation Award for his work on data mining and Research Division Award for his work on object-oriented databases. His current research interests include data mining, text and web mining, OLAP, and electronic commerce. He is a senior member of IEEE. 